Ergod. Th. \& Dynam. Sys. (First published online 2006), 0, 1-35*

doi:10.1017/S0143385706000289 (c) 2006 Cambridge University Press

*Provisional-final page numbers to be inserted when paper edition is published

\title{
Catastrophe theory in Dulac unfoldings
}

\author{
H. W. BROER $\dagger$, V. NAUDOT $\dagger$ and R. ROUSSARIE $\ddagger$ \\ $\dagger$ Department of Mathematics and Computing Science, University of Groningen, \\ Blauwborgje 3, 9747 AC Groningen, The Netherlands \\ (e-mail:vijnc@maths.warwick.ac.uk) \\ †Institut Mathématiques de Bourgogne, CNRS, 9 avenue Alain Savary, B.P. 47 870, \\ 21078 Dijon Cedex, France
}

(Received 8 December 2004 and accepted in revised form 13 April 2006)

\begin{abstract}
In this paper we study analytic properties of compensator and Dulac expansions in a single variable. A Dulac expansion is formed by monomial terms that may contain a specific logarithmic factor. In compensator expansions this logarithmic factor is deformed. We first consider Dulac expansions when the power of the logarithm is either 0 or 1 . Here we construct an explicit exponential scaling in the space of coefficients which in an exponentially narrow horn, up to rescaling and division, leads to a polynomial expansion. A similar result holds for the compensator case. This result is applied to the bifurcation theory of limit cycles in planar vector fields. The setting consists of families that unfold a given Hamiltonian in a dissipative way. This leading part is of Morse type, which leads to the following three cases. The first concerns a Hamiltonian function that is regular on an annulus, and the second a Hamiltonian function with a non-degenerate minimum defined on a disc. In the third case the Hamiltonian function has a non-degenerate saddle point with a saddle connection. The first case, by an appropriate scaling, recovers the generic theory of the saddle node of limit cycles and its cuspoid degeneracies, while the second case similarly recovers the generic theory of limit cycles subordinate to the codimension $k$ Hopf bifurcations $k=1,2, \ldots$. The third case enables a novel study of generic bifurcations of limit cycles subordinate to homoclinic bifurcations. We then describe how the above analytic result is applied to bifurcations of limit cycles. For appropriate one-dimensional Poincaré maps, the fixed points correspond to the limit cycles. The fixed point sets (or zero-sets of the associated displacement functions) are studied by contact equivalence singularity theory. The cases where the Hamiltonian is defined on an annulus or a disc can be reduced directly to catastrophe theory. In the third case, the displacement functions are known to have compensator expansions, whose first approximations are Dulac expansions. Application of our analytic result implies that, in an exponentially narrow horn near a homoclinic loop, the bifucation theory of limit cycles again reduces to catastrophe theory.
\end{abstract}

\section{Introduction}

This paper contributes to the study of generic families of planar diffeomorphisms near a degenerate fixed point $[\mathbf{3}, \mathbf{1 5}, \mathbf{4 7 - 4 9}]$. Note that such diffeomorphisms can be obtained 
from time-periodic planar vector fields, by taking a Poincaré (return) map. Usually, by normal form or averaging techniques $[\mathbf{3}, \mathbf{1 7}, \mathbf{4 0}]$, the family becomes autonomous to a large order. In many cases, after suitable rescaling the family is the sum of a Hamiltonian vector field that does not depend on the parameters, a dissipative part and a non-autonomous part. The latter part consists of higher-order terms in the rescaling parameter [5, 23-25, 47]. This approach covers many generic cases like the codimension $k$ Hopf bifurcations including degenerate Hopf (or Chenciner) and Hopf-Takens bifurcations [15, 20-22, 46-49] but also the Bogdanov-Takens bifurcation for maps $[4,16]$. Our interest is with the geometry of the bifurcation set of limit cycles of the autonomous system, which correspond to invariant tori in the full system. The latter were studied systematically by Broer et al [12]. Limit cycles of the autonomous system correspond to fixed points of the Poincaré map (or zeros of the displacement function) on one-dimensional sections. For values of the parameter that are relatively far from the bifurcation set of limit cycles associated to the autonomous system, the dynamics of the non-autonomous system is of Morse-Smale type [41]. The genericity conditions on the original family often lead to certain explicit genericity conditions on the corresponding family of scaled displacement functions $[\mathbf{1 4}, \mathbf{1 9}, \mathbf{2 3}, \mathbf{2 4}$, which in our set-up will be translated to suitable hypotheses. By the division theorem, the latter lead to structural stability of a scaled displacement function under contact equivalence $[30,32,39]$.

The starting point of the present paper will be the family of non-autonomous systems after rescaling, although our results are concerned with bifurcations of limit cycles in the autonomous truncation. In Example 1 below, we show the classical way in which this format is obtained; later on, more classes of examples will be discussed. Before that, we note that for the full non-autonomous family, the autonomous bifurcation diagram provides a skeleton indicating the complexity of the actual dynamics [20, 50]. However, as Broer and Roussarie [14] have shown, this dynamical complexity near the bifurcation set of limit cycles, in the real analytic setting, is confined to an exponentially narrow horn. For earlier results in this direction, see [12].

Example 1. (Bogdanov-Takens bifurcation) [4, 6, 17, 25] Consider the following unfolding of the codimension two bifurcation

$$
X_{\mu, v}=y \frac{\partial}{\partial x}+\left(x^{2}+\mu+y v \pm y x+R(x, y, t, \mu, v)\right) \frac{\partial}{\partial y},
$$

where $R$ is $2 \pi$-periodic in the time $t$ and represents the non-autonomous part. We assume that $|R(x, y, t, \mu, v)|=\mathcal{O}\left(x^{2}+y^{2}\right)^{3 / 2}$. Under the classical rescaling $[\mathbf{4}, \mathbf{6}]$

$$
x=\varepsilon^{2} \bar{x}, \quad y=\varepsilon^{3} \bar{y}, \quad \mu=-\varepsilon^{4}, \quad v=\varepsilon^{2} \bar{v},
$$

the system above becomes

$$
\varepsilon \bar{Y}_{\varepsilon, \bar{v}}=\varepsilon \bar{y} \frac{\partial}{\partial \bar{x}}+\varepsilon\left(\left(\bar{x}^{2}-1\right)+\varepsilon \bar{y} \bar{v} \pm \varepsilon \bar{y} \bar{x}+\mathcal{O}\left(\varepsilon^{3}\right)\right) \frac{\partial}{\partial \bar{y}} .
$$

We see that $\bar{Y}_{\varepsilon, \bar{v}}=X_{H}+\varepsilon Z_{\varepsilon, \bar{v}}+\mathcal{O}\left(\varepsilon^{2}\right)$, where

$$
X_{H}=\bar{y} \frac{\partial}{\partial \bar{x}}+\left(\bar{x}^{2}-1\right) \frac{\partial}{\partial \bar{y}}
$$


is a Hamiltonian that does not depend on the parameters $(\bar{v}, \varepsilon)$ and possesses a homoclinic loop to a hyperbolic saddle. The loop encloses a disc containing a centre. Notice that the non-autonomous terms are included in the $\mathcal{O}\left(\varepsilon^{3}\right)$ part. See $[\mathbf{6}, \mathbf{1 4}]$ for details.

This example motivates the general perturbation setting of our paper. More examples will be recalled below $[\mathbf{6}, \mathbf{1 5}, \mathbf{1 7}, \mathbf{2 3}, \mathbf{2 4}]$ and certain new examples will be treated in detail. Our focus will be on bifurcations of limit cycles near a homoclinic trajectory of the Hamiltonian vector field. By an explicit exponential scaling in the parameter space, an exponentially narrow horn is created, in which the bifurcation set of limit cycles can be completely described. Indeed, the structural stability of a scaled displacement function under contact equivalence leads to the full complexity of catastrophe theory. For the original autonomous family of vector fields, inside the horn, this yields structural stability under weak orbital equivalence $[\mathbf{5}, \mathbf{2 6}, \mathbf{2 7}]$, where the reparametrizations are $C^{\infty}$. In summary, our results reveal an exponentially narrow fine structure of the parameter space, that regards the dynamics of the autonomous family of planar vector fields. We expect that our results will be of help when studying the full system on the solid torus $\mathbb{R}^{2} \times \mathbb{S}^{1}$, where the 'unperturbed' limit cycles correspond to invariant 2-tori with parallel dynamics. When turning to the non-autonomous 'perturbed' system, persistence of these tori becomes a matter of KAM theory, in particular of quasi-periodic bifurcation theory $[\mathbf{5 , 1 1}]$. Indeed, the 2-tori with Diophantine frequencies form a Cantor foliation of hypersurfaces in the parameter space, and we conjecture that most of the Diophantine tori persist, including their bifurcation pattern. The KAM persistence results involve a Whitney smooth reparametrization which is near the identity map in terms of $|\varepsilon| \ll 1$. This means that the perturbed Diophantine 2-tori correspond to a perturbed Cantor foliation in the parameter space. In the complement of this perturbed Cantor foliation of hypersurfaces, we expect all the dynamical complexity regarding Cantori, strange attractors, etc., as described in [19-22, 38, 43].

1.1. Background and setting of the problem. The general perturbation format of the present paper is given by the family of $C^{\infty}$ planar, time-periodic vector fields

$$
X_{\lambda, \varepsilon}+\varepsilon^{r} R \quad \text { with } X_{\lambda, \varepsilon}=\varepsilon^{p} X_{H}+\varepsilon^{q} Y_{\lambda, \varepsilon}
$$

where $X_{\lambda, \varepsilon}$ consists of the autonomous part. The following properties hold. First $0 \leq p<$ $q<r$ are integers. Secondly, $\lambda \in \mathbb{R}^{\ell}$ is a (multi)parameter varying near 0 , while $\varepsilon>0$ is a perturbation parameter. Thirdly, the vector field $R$ is $2 \pi$-periodic in the time $t$, and may depend on all the other variables and parameters as well. Finally, the vector field $X_{H}$ is a Hamiltonian and does not depend on the parameters. Notice that, in Example 1, we have $p=1, q=2$ and $r=3$.

We begin by discussing the Hamiltonian function $H$. Our genericity setting implies that $H$ should be a stable Morse function, which leaves us with three possible cases $X_{H}$ is defined in an annulus, near a centre or near a homoclinic loop (see Figure 1). In each case, we can define a Poincaré return map

$$
\mathcal{P}_{\lambda, \varepsilon}: \Sigma_{0} \rightarrow \Sigma \quad \text { with } \mathcal{P}_{\lambda, \varepsilon}(x)=x+\varepsilon^{q-p} B_{\lambda, \varepsilon}(x)
$$




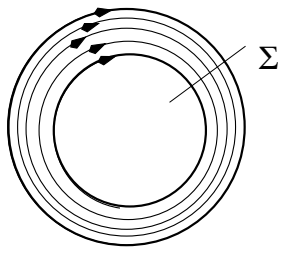

(a)

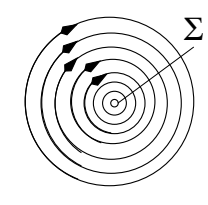

(b)

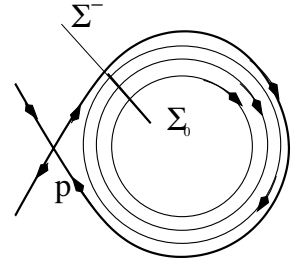

(c)

Figure 1. The Hamiltonian $X_{H}$ in an annulus (a), near a centre (b), and near a homoclinic loop (c).

Here $\Sigma_{0} \subset \Sigma$ is a transversal section and $B_{\lambda, \varepsilon}(x)$ is the scaled displacement function. Limit cycles are in a one-to-one correspondence with the zeros of $B_{\lambda, \varepsilon}$. The bifurcation set $\mathcal{B}$ of limit cycles is defined as

$$
\mathcal{B}=\left\{(\lambda, \varepsilon) \in \mathbb{R}^{\ell} \times \mathbb{R}^{+} \mid B_{\lambda, \varepsilon}(x)=B_{\lambda, \varepsilon}^{\prime}(x)=0 \text { for some } x \in \Sigma_{0}\right\} .
$$

Denote $\mathcal{B}_{\varepsilon_{0}}=\mathcal{B} \cap\left\{\varepsilon=\varepsilon_{0}\right\}$. The family $Y_{\lambda, \varepsilon}$ is generic in a sense to be made precise below and which by the division theorem $[27,32,36,39]$ implies $C^{\infty}$ structural stability under contact equivalence of the scaled displacement function $B_{\lambda, \varepsilon}$ in the following sense. In the following definition the families of functions are local, i.e. we consider germs of families.

Definition 1. (Contact equivalence) $[\mathbf{5}, \mathbf{2 6}, \mathbf{3 6}, \mathbf{4 3}, \mathbf{4 9}]$ Let $f_{\lambda}:(\mathbb{R}, 0) \rightarrow(\mathbb{R}, 0)$ and $g_{\mu}:(\mathbb{R}, 0) \rightarrow(\mathbb{R}, 0)$ be two families of functions, with $\lambda, \mu \in\left(\mathbb{R}^{\ell}, 0\right)$. These families are $\mathcal{C}^{\infty}$-contact equivalent if there exist a $C^{\infty}$ diffeomorphism $\varphi:\left(\mathbb{R}^{\ell}, 0\right) \rightarrow\left(\mathbb{R}^{\ell}, 0\right)$, a $C^{\infty}$ family of diffeomorphisms $h_{\lambda}:(\mathbb{R}, 0) \rightarrow(\mathbb{R}, 0)$ and a $C^{\infty}$ function $U:(\mathbb{R}, 0) \rightarrow(\mathbb{R}, 0)$ with $U(0) \neq 0$ such that

$$
f_{\lambda} \circ h_{\lambda}(u)=U(u) \cdot g_{\varphi(\lambda)}(u) .
$$

The definition implies that $h_{\lambda}$ sends the zero-set of $f_{\lambda}$ to the zero-set of $g_{\varphi(\lambda)}$ preserving multiplicity. As a consequence, for small values of $\varepsilon$, the zero-set $\mathcal{B}_{\varepsilon}$ is diffeomorphically equivalent to $\mathcal{B}_{0}$. Although our main interest is with the homoclinic loop case, we first briefly revisit the simpler cases of an annulus and a centre.

1.1.1. Classical cases of polynomial geometry. In the case of an annulus (see Figure 1(a)), by our assumption of structural stability under contact equivalence and by the division theorem $[\mathbf{2 7}, \mathbf{3 2}, \mathbf{3 6}, \mathbf{3 9}], B_{\lambda, \varepsilon}$ takes the form

$$
B_{\lambda, \varepsilon}(x)=U(x, \lambda, \varepsilon) Q_{k}^{s, \pm}(x, \beta(\lambda, \varepsilon)) .
$$

In (3), $U \neq 0$ is smooth and

$$
Q_{k}^{s, \pm}(x, \beta(\lambda, \varepsilon))=\beta_{0}(\lambda, \varepsilon)+\beta_{1}(\lambda, \varepsilon) x+\cdots+\beta_{k-2}(\lambda, \varepsilon) x^{k-2} \pm x^{k},
$$

which is the standard catastrophe cuspoid normal form. Here, the map

$$
\lambda \mapsto\left(\beta_{0}(\lambda, 0), \ldots, \beta_{k-2}(\lambda, 0)\right)
$$


is a submersion. The codimension of the singularity is $k-1$. For $\varepsilon$ sufficiently small, the bifurcation set $\mathcal{B}_{\varepsilon}$ consists of all $\lambda$-values such that

$$
Q_{k}^{s, \pm}(x, \beta(\lambda, \varepsilon))=\frac{d Q_{k}^{s, \pm}}{d x}(x, \beta(\lambda, \varepsilon))=0,
$$

for some $x$ near 0 . Since $Q_{k}^{s, \pm}$ takes the form (4), we say that, for sufficiently small $\varepsilon$, the geometry of the bifurcation set of limit cycles $\mathcal{B}_{\varepsilon}$ is polynomial. By studying the zero-set $\mathcal{B}_{0}$ using standard catastrophe theory $[\mathbf{5}, \mathbf{2 9}, \mathbf{4 3}, \mathbf{5 3}]$, we recover the generic bifurcation theory of limit cycles as it now has become standard $[\mathbf{5 , 2 6 , 2 9 ]}$. For instance, in the case $k=2$, the bifurcation set is called a fold corresponding to a saddle node of limit cycles; in the case $k=3$ one obtains a cusp of limit cycles.

In the case of a centre (see Figure 1(b)), by similar arguments, the reduced displacement function takes the form

$$
B_{\lambda, \varepsilon}(u)=U(u, \lambda, \varepsilon) Q_{k}^{p, \pm}(u, \alpha(\lambda)),
$$

where $u=r^{2}, r$ being the distance to the origin, with the pointed catastrophe cuspoid normal form

$$
Q_{k}^{p, \pm}(u, \alpha)=\alpha_{0}(\lambda, \varepsilon)+\alpha_{1}(\lambda, \varepsilon) u+\cdots+\alpha_{k-1}(\lambda, \varepsilon) u^{k-1} \pm u^{k}, \quad u \geq 0 .
$$

We remark that $Q_{k}^{p, \pm}$ possesses a term of order $k-1$ while $Q_{k}^{s, \pm}$ does not. For more details, see [14]. Again, the map

$$
\lambda \mapsto\left(\alpha_{0}(\lambda, 0), \ldots, \alpha_{k-1}(\lambda, 0)\right)
$$

is a submersion and the codimension of the singularity is $k$. For each $\varepsilon$ sufficiently small, the set $\mathcal{B}_{\varepsilon}$ consists of all $\lambda$-values such that

$$
Q_{k}^{p, \pm}(u, \alpha(\lambda, \varepsilon))=\frac{d Q_{k}^{p, \pm}}{d u}(u, \alpha(\lambda, \varepsilon))=0,
$$

for some $u>0$. As in the annulus case, for each sufficiently small $\varepsilon$, since $Q_{k}^{p, \pm}$ takes the form (5), the geometry of the bifurcation set of limit cycles $\mathcal{B}_{\varepsilon}$ is polynomial. Now $\mathcal{B}_{\varepsilon}$ is described by the pointed catastrophe theory. This set-up recovers all codimension $k$ Hopf bifurcations $[14,19,47]$.

1.1.2. The homoclinic loop case. Our main interest in the present paper is with the case where the Hamiltonian $H$ possesses a homoclinic loop $\Gamma$ (see Figure 1(c)). Here the Poincaré return map is defined on a half section $\Sigma_{0} \subset \Sigma$ parametrized by $u \geq 0$. The difference between the present and the above cases is that the displacement function is singular at $u=0$ and so cannot be written as a Taylor expansion at this value. When $\varepsilon=0$, the displacement function takes the form of a Dulac expansion $[\mathbf{1}, \mathbf{3 4}, \mathbf{3 5}, \mathbf{4 4}]$; and when $\varepsilon \neq 0$, it takes the form of a compensator expansion $[\mathbf{3 4}, \mathbf{3 5}, \mathbf{4 4}]$, which deforms the Dulac expansion. We shall be more precise in the next section.

The aim of this paper is to present a division theorem which is adapted to Dulac and compensator unfoldings. We shall construct a singular scaling in the parameter space so 
that the corresponding family of displacement functions becomes structurally stable under contact equivalence, and, thanks to the classical division theorem, takes the form (3). This implies that the bifurcation set $\mathcal{B}_{\varepsilon}$ has polynomial geometry. The function $U$ in (3), although smooth in the interior of the horn, contains all non-regularity of the displacement function, i.e. has Dulac asymptotics at the tip of the horn. The region in the phase space to be considered, though arbitrarily close to $\Gamma$, does not contain $\Gamma$. However, the region does contain all the (bifurcating) limit cycles.

1.1.3. Example: the codimension two saddle connection. To fix thoughts, before stating our results, we announce an application in the case of a codimension two degenerate homoclinic orbit $[\mathbf{1 4}, \mathbf{2 3}, \mathbf{2 4}]$, as it is subordinate to a degenerate Bogdanov-Takens bifurcation.

Example 2. (Degenerate Bogdanov-Takens bifurcation) $[\mathbf{1 4}, \mathbf{2 3}, \mathbf{2 4}]$ Consider the following unfolding of the codimension three Bogdanov-Takens bifurcation

$$
X_{\mu, v_{0}, v_{1}}=y \frac{\partial}{\partial x}+\left(x^{2}+\mu+y v_{0}+v_{1} y x \pm y x^{3}\right) \frac{\partial}{\partial y} .
$$

This family is studied in $[\mathbf{2 3}, \mathbf{2 4}]$. The bifurcation set is a topological cone with vertex at $0 \in \mathbb{R}^{3}$ that is transverse to the 2-sphere $\mathbb{S}^{2}$. The trace of the bifurcation set on $\mathbb{S}^{2}$ possesses, among various bifurcation points of codimension two, a point of degenerate saddle connection. After suitable rescaling [14], the family here takes the form

$$
X_{\mu, \bar{v}_{0}, \bar{v}_{1}}=\varepsilon \bar{y} \frac{\partial}{\partial \bar{x}}+\varepsilon\left(\bar{x}^{2}-1+\varepsilon^{5}\left(\bar{y} \bar{v}_{0}+\bar{v}_{1} \bar{y} \bar{x} \pm \bar{y} \bar{x}^{3}\right)\right) \frac{\partial}{\partial \bar{y}} .
$$

In terms of our general set-up, the family takes the form (1) with $p=1, q=6$ and

$$
H(\bar{x}, \bar{y})=\frac{1}{2} \bar{y}^{2}-\frac{1}{3} \bar{x}^{3}+\bar{x} .
$$

The phase portrait of $X_{H}$ is described in Figure 1(c). The scaled displacement function takes the form

$$
B_{v, \varepsilon}(u)=\alpha_{0}\left(\nu_{0}, \nu_{1}, \varepsilon\right)+\beta_{1}\left(\nu_{0}, \nu_{1}, \varepsilon\right) u \omega_{\varepsilon}(u)+c u+o(u),
$$

where $\omega_{\varepsilon}(u)$ is a compensator function $[\mathbf{1 5}, \mathbf{1 8 , 3 4 - 3 6}]$ and where $\omega_{0}(u)=\log u$, where $u$ is the distance to the saddle connection. In this example, it is generic to assume that $c \neq 0$ and that the map

$$
\left(\nu_{0}, v_{1}\right) \mapsto\left(\alpha_{0}\left(v_{0}, v_{1}, 0\right), \beta_{1}\left(v_{0}, v_{1}, 0\right)\right)
$$

is a local diffeomorphism near 0 . For simplicity, we restrict to the case $\varepsilon=0$, since the case $\varepsilon \neq 0$ is just more complicated without being qualitatively different. Theorem 2 gives the scaling

$$
\Psi:(-A, A) \times\left(0, \tau_{0}\right) \rightarrow \mathbb{R}^{2}, \quad\left(\gamma_{0}, \tau\right) \mapsto\left(\alpha_{0}, \beta_{1}\right),
$$

where $A>0, \tau_{0}>0$ and where

$$
\begin{gathered}
\beta_{1}=-\frac{c+\tau \log \tau Q_{1}(\tau)}{1+\log \tau}, \\
\alpha_{0}=\frac{\gamma_{0} \tau}{1+\log \tau}-\frac{-c \tau+\tau^{2} \log \tau R_{0}(\tau)}{1+\log \tau},
\end{gathered}
$$




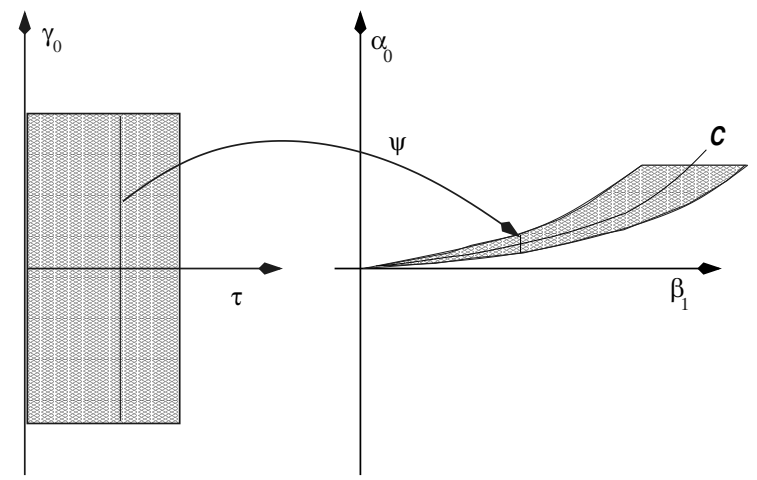

FIGURE 2. Image of a rectangle $(-A, A) \times\left(0, \tau_{0}\right)$ under the scaling $\Psi$. On the right-hand side the corresponding region is an exponentially flat horn. The resulting bifurcation set $\mathcal{B}_{0}$, located in the dashed region on the righthand side, is the product of a fold bifurcation set and an interval.

where $Q_{1}$ and $R_{0}$ are $C^{\infty}$ (see Figure 2). We claim that as an application of our results,

$$
B_{\Psi\left(\gamma_{0}, \tau\right), 0}(\tau(1+x))=\frac{\tau}{1+\log \tau}\left(\gamma_{0}-\left(\frac{1}{2}+\mathcal{O}(\tau \log \tau)\right) x^{2}+\mathcal{O}\left(x^{3}\right)\right),
$$

and by the division theorem, we have

$$
B_{\Psi\left(\gamma_{0}, \tau\right), 0}(\tau(1+x))=\frac{\tau}{1+\log \tau} \tilde{U}\left(x, \gamma_{0}, \tau\right)\left(\gamma_{0}-\frac{x^{2}}{2}\right),
$$

where $\tilde{U}$ is $C^{\infty}$ and has Dulac asymptotics at $\tau=0$, see below. Therefore

$$
B_{\Psi\left(\gamma_{0}, \tau\right), 0}(\tau(1+x))=U\left(x, \gamma_{0}, \tau\right)\left(\gamma_{0}-x^{2}\right),
$$

where

$$
U\left(x, \gamma_{0}, \tau\right)=\frac{\tau}{1+\log \tau} \tilde{U}\left(x, \gamma_{0}, \tau\right)
$$

see Definition 1.

The idea of the scaling in (7) is the following. Observe that the form (6) contains a logarithmic term and therefore is singular at $u=0$. However, the form is no longer singular at $u=\tau$ for any $\tau>0$ arbitrarily small. By putting $u=\tau(1+x)$, we localize the study of (7) near $u=\tau$, i.e. near $x=0$. The map

$$
\Phi_{\Psi\left(\gamma_{0}, \tau\right)}(x)=B_{\Psi\left(\gamma_{0}, \tau\right), 0}(\tau(1+x))
$$

is regular and can be expanded in a Taylor series. All non-regularity of the unfolding is now contained in the parameter dependence (7).

From (7), we have

$$
\alpha_{0} \sim-\left(\gamma_{0}-c\right) \beta_{1} \frac{e^{-1 / \beta_{1}}}{e}
$$

Since $\gamma_{0}$ belongs to an interval of length $2 A$, for fixed $\beta_{1}$, we have the following exponential estimate:

$$
\alpha_{0} \sim \frac{2 A \beta_{1}}{e} e^{-1 / \beta_{1}}, \quad \beta_{1}>0
$$


The bifurcation set of limit cycles is given by $\mathcal{B}_{0}=\Psi(\mathcal{F})$ where

$$
\mathcal{F}=\left\{\gamma_{0}=0\right\},
$$

which is the product of a fold and an interval and corresponds to a saddle node bifurcation of limit cycles.

As a consequence of the estimate (9), the image of the rescaling is an exponentially flat region (see Figure 2). Observe that the set of homoclinic bifurcation which is given by $\mathcal{H}=\left\{\alpha_{0}=0\right\}$ is contained in the image of the rescaling introduced in (7). However, since the study of the displacement function is localized near $u=\tau \ll \exp \left(-1 /\left|\beta_{1}\right|\right)$, the region of the phase space under consideration is an exponentially narrow annulus, which does not contain the homoclinic orbit. Therefore, the subordinate homoclinic bifurcation cannot be studied with the help of the form (8). Up to division by $U$, the displacement function is polynomial; indeed, it is the normal form of a fold $[43,53]$.

As mentioned before, the stability of the displacement function under contact equivalence implies weak orbital stability of the vector field unfolding with $C^{\infty}$ reparametrization.

Remark. In Example 2, we find a saddle node of limit cycles inside the horn, corresponding to a fold catastrophe of the scaled displacement function. Using similar techniques, stable limit cycles are discovered in $[\mathbf{1 4}, \mathbf{1 6}]$ that correspond to scaled displacement functions of Morse type.

1.1.4. Outline. This paper is organized as follows. All results will be formulated in $\S 1.2$. We first study the rescaled displacement function $B_{\lambda, \varepsilon}$ when $\varepsilon=0$, in which case $B_{\lambda, 0}$ has a Dulac expansion. In Theorems 1 and 2 we present normal forms for $B_{\lambda, 0}$, showing that the geometry of its zero-set again is polynomial. Also we give an explicit algorithm for the normalizing rescaling. We mention that Theorem 2 generalizes the example of $\S 1.1 .3$, extending it to all cuspoid bifurcations of limit cycles of even degree. Second we treat the case $\varepsilon>0$, in which the scaled displacement function will have a socalled compensator expansion, which deforms the above Dulac case. Theorems 3 and 4 are the analogues of Theorems 1 and 2 for this compensator case.

Section 1.3 contains an example concerning a cusp bifurcation of limit cycles, as it is subordinate to a codimension four Bogdanov-Takens bifurcation [31, 34, 35]. This example is an application of Theorem 1. In $\S 1.4$ we discuss further potential applications of our results for the full 'perturbed' system, where our statements are mainly conjectural and pointing to future research. All proofs are postponed to $\S 2$.

1.2. Results. We briefly recall the general setting. The family of planar autonomous vector fields to be considered has the form (1),

$$
X_{\lambda, \varepsilon}=\varepsilon^{p} X_{H}+\varepsilon^{q} Y_{\lambda, \varepsilon},
$$

where $X_{H}$ possesses a homoclinic orbit $\Gamma \subset\{H=0\}$. We focus our study near this homoclinic loop. For all $0<u<u_{0}$ where $u_{0}$ is close to 0 , orbits of $X_{H}$ contained in $\Gamma_{u} \subseteq\{H=u\}$ are supposed to be periodic, $\Gamma_{u}$ tending to the homoclinic loop $\Gamma$ 
as $u$ tends to 0 in the Hausdorff topology. The subsection $\Sigma_{0} \subset \Sigma$ (see Figure 1), on which the Poincaré return map is well defined, transversally intersects each of these periodic orbits. The Poincaré return map takes the form (2). The asymptotics of $B_{\lambda, \varepsilon}$ is given by Roussarie [44]. For convenience, we introduce the 1 -form $\iota_{X, \varepsilon} \Omega$, which is dual to the vector field $X_{\lambda, \varepsilon}$ by the standard area form $\Omega$ on $\mathbb{R}^{2}$, i.e.

$$
\iota_{X_{\lambda, \varepsilon}} \Omega=\varepsilon^{p} d H+\varepsilon^{q} \omega_{\lambda, 0}+o(\varepsilon) .
$$

We have

$$
B_{\lambda, 0}(u)=\int_{\Gamma_{u}} \omega_{\lambda, 0} .
$$

This Abelian integral expands on a logarithmic scale, to be described below. Such an expansion is called a Dulac expansion. In Theorems 1 and 2 a scaling in the parameter space

$$
\Psi: \mathbb{R}^{k-1} \times \mathbb{R}^{+} \rightarrow \mathbb{R}^{k}, \quad(\gamma, \tau) \mapsto \Psi(\gamma, \tau)
$$

is constructed in such a way that

$$
B_{\Psi(\gamma, \tau), 0}(\tau(1+x))=\tilde{f}(\tau)\left(\gamma_{0}+\gamma_{1} x+\cdots+\gamma_{k-2} x^{k-2}+k(\gamma, \tau) x^{k}+\mathcal{O}\left(x^{k+1}\right)\right),
$$

where $\tilde{f}(\tau)=\tau^{n} \log \tau$, in the case $k=2 n-1$, and $\tilde{f}(\tau)=\tau^{n} /(1+\log \tau)$, in the case $k=2 n$, and where $k(0) \neq 0$. The idea behind the construction of the scaling $\Psi$ is the following. By putting $u=\tau(1+x)$, the map

$$
\Phi_{\alpha, \beta}: \mathbb{R} \rightarrow \mathbb{R}, \quad x \mapsto B_{\alpha, \beta, 0}(\tau(1+x))
$$

becomes $C^{\infty}$ at the origin, and we expand $\Phi_{\alpha, \beta}$ in a Taylor series. In the generic case, we may identify the coefficients of the Dulac expansion with $\lambda$. First, in the $(\alpha, \beta)$-space, a parametrization of the curve $\mathcal{C}$ by $\tau$ is given by

$$
\mathcal{C}=\left\{(\alpha, \beta) \in \mathbb{R}^{d} \mid \Phi_{\alpha, \beta}(0)=\Phi_{\alpha, \beta}^{\prime}(0)=\cdots=\Phi_{\alpha, \beta}^{(k-1)}(0)=0, \Phi_{\alpha, \beta}^{(k)}(0) \neq 0\right\} .
$$

This latter curve emanating from the origin is of 'highest degeneracy', i.e. along this curve

$$
\Phi_{\Psi(0, \tau)}(x)=\tilde{f}(\tau)\left(k(0, \tau) x^{k}+\mathcal{O}\left(x^{k+1}\right)\right) .
$$

Secondly, the scaling $\Psi$ is defined. The image of $\Psi$ is a narrow neighbourhood of $\mathcal{C}$ and, for each parameter value in this neighbourhood, the map $\Phi_{\Psi(\gamma, \tau)}$ takes the form (11). By the division theorem $[27,32,36,39]$ applied to $(11)$, we obtain

$$
\begin{aligned}
\Phi_{\Psi(\gamma, \tau)}(x) & =\tilde{U}(x, \gamma, \tau) \tilde{f}(\tau)\left(\gamma_{0}+\gamma_{1} x+\cdots+\gamma_{k-2} x^{k-2} \pm x^{k}\right) \\
& =U(x, \tau, \gamma) Q_{k}^{s, \pm}(x, \gamma),
\end{aligned}
$$

where $U(x, \tau, \gamma)=\tilde{f}(\tau) \tilde{U}(x, \gamma, \tau)$. All non-regularity of the unfolding is hidden in the function $U$, and after division by this latter, the displacement function is polynomial. In a final step, we consider the complete compensator expansion given in [44]. In Theorem 3, respectively Theorem 4 , we show that there exists an analytic deformation of the scaling proposed in Theorem 1, respectively Theorem 2, such that $B_{\Psi(\gamma, \tau), \varepsilon}$ again takes the form (11). 
1.2.1. The Dulac case. We now give a precise description of the Dulac case. The logarithmic scale of functions is given by

$$
\mathcal{L}=\left\{1, u \log u, u, \ldots, u^{i}, u^{i+1} \log u, \ldots\right\} .
$$

The Abelian integral (10) expands on this scale [34-36, 45]. This means that there exist sequences of $C^{\infty}$ functions $\alpha_{i}(\lambda)$ and $\beta_{j}(\lambda)$ for integers $i \geq 0$ and $j \geq 1$ such that

$$
B_{\lambda, 0}(u)=\sum_{i=0}^{N} \alpha_{i} u^{i}+\sum_{j=1}^{N} \beta_{j} u^{j} \log u+o\left(u^{N}\right),
$$

for any $N \in \mathbb{N}$.

We say that (12) is a non-degenerate Dulac unfolding of order $2 n$ if $\alpha_{n} \neq 0$ but for each integer $i=0, \ldots, n-1$ one has $\beta_{i+1}(0)=0=\alpha_{i}(0)$ and if moreover the map

$$
\lambda \mapsto\left(\alpha_{0}(\lambda), \beta_{1}(\lambda), \ldots, \alpha_{n-1}(\lambda), \beta_{n}(\lambda)\right)
$$

is a submersion. In this case, we rewrite (12) as

$$
B_{\lambda, 0}(u)=\sum_{i=0}^{n-1} \alpha_{i}(\lambda) u^{i}+\sum_{i=1}^{n} \beta_{i}(\lambda) u^{i} \log u+c u^{n}+o\left(u^{n}\right)
$$

where $c=\alpha_{n}(\lambda)$.

Similarly we say that (12) is a non-degenerate Dulac unfolding of codimension $2 n-1$ if $\beta_{n}(0) \neq 0$ but $\alpha_{0}(0)=0$ and for each integer $i=1, \ldots, n-1$ one has $\beta_{i}(0)=0=\alpha_{i}(0)$ and if moreover the map

$$
\lambda \mapsto\left(\alpha_{0}(\lambda), \beta_{1}(\lambda), \ldots, \alpha_{n-2}(\lambda), \beta_{n-1}(\lambda), \alpha_{n-1}(\lambda)\right)
$$

is a submersion. In this case, we rewrite (12) as

$$
B_{\lambda, 0}(u)=\sum_{i=0}^{n-1} \alpha_{i}(\lambda) u^{i}+\sum_{i=1}^{n-1} \beta_{i}(\lambda) u^{i} \log u+c u^{n} \log ^{n}+\mathcal{O}\left(u^{n}\right),
$$

where $c=\beta_{n}(\lambda)$. We introduce the following notation. Let $F:(\mathbb{R}, 0) \rightarrow \mathbb{R}$ be a Dulac expansion of the form (12). We then write

$$
F(u)=c u^{m} \log ^{\ell}(u)+\cdots=c u^{m} \log ^{\ell}(u)+\sum_{(m, \ell) \ll(k, j)} c_{\ell, j} u^{k} \log ^{j}(u),
$$

where the order $\ll$ on the set

$$
\mathbb{N}^{s}=\{(a, b) \in \mathbb{N} \times \mathbb{Z} \mid b \leq a\},
$$

is defined as

$$
(\ell, j) \ll(\ell+1, k) \text { for all } j \leq \ell, k \leq \ell+1 \text {, and }(\ell, j-1) \ll(\ell, j) .
$$

In what follows $\mathbb{R}_{+}$denotes the set of strictly positive real numbers. We now state the first results. 
THEOREM 1. (Dulac case of odd codimension) Let

$$
\begin{gathered}
B: \mathbb{R}_{+} \times \mathbb{R}^{n} \times \mathbb{R}^{n-1} \rightarrow \mathbb{R}, \\
(u, \alpha, \beta) \mapsto B_{\alpha, \beta}(u)=\sum_{i=0}^{n-1} \alpha_{i} u^{i}+\sum_{i=1}^{n-1} \beta_{i} u^{i} \log u+c\left[u^{n} \log u+\cdots\right],
\end{gathered}
$$

be a non-degenerate Dulac unfolding of codimension $2 n-1$. Then

$$
B_{\Psi(\gamma, \tau)}(\tau(1+x))=k(\gamma, \tau) \tau^{n} \log \tau\left(Q_{2 n-1}^{s, \pm}(x, \gamma)+\mathcal{O}\left(x^{2 n}\right)\right),
$$

where $k(\gamma, \tau) \neq 0$ depends $C^{\infty}$ on $(\gamma, \tau)$, and where

$$
\Psi: \mathbb{R}^{2 n-2} \times \mathbb{R}_{+} \rightarrow \mathbb{R}^{2 n-1}, \quad(\gamma, \tau) \mapsto(\alpha(\gamma, \tau), \beta(\gamma, \tau)),
$$

gives the change of parameters. The map $\Psi$ is $C^{\infty}$ and of the form

$$
\begin{aligned}
\alpha_{i} & =\tau^{n-i} \log ^{2} \tau \bar{\alpha}_{i}(\gamma, \tau), & & 0 \leq i \leq n-1, \\
\beta_{i} & =\tau^{n-i} \log \tau \bar{\beta}_{i}(\gamma, \tau), & & 1 \leq i \leq n-1 .
\end{aligned}
$$

We further specify

$$
\begin{gathered}
\bar{\beta}_{i}(\gamma, \tau)=-c a_{i}+\mathbb{V}_{i}(\gamma)-\log ^{-1} \tau \bar{R}_{n-1+i}(\tau) \\
\bar{\alpha}_{n-1}(\gamma, \tau)=-\sum_{j=1}^{n-1}\left(L_{n-1, j} \log ^{-1} \tau+H_{n-1, j}\right) \bar{\beta}_{j}(\gamma, \tau)+d_{n-1} \log ^{-1} \tau \\
+\gamma_{n-1} \log ^{-1} \tau-\left(c L_{n-1, n}+\bar{P}_{n-1}(\tau)\right) \log ^{-2} \tau
\end{gathered}
$$

and for each integer $i=0, \ldots, n-2$,

$$
\begin{aligned}
\bar{\alpha}_{i}(\gamma, \tau)= & -\sum_{j=i+1}^{n-1} J_{i, j} \bar{\alpha}_{j}(\gamma, \tau)-\sum_{j=1}^{n-1}\left(L_{i, j} \log ^{-1} \tau+H_{i, j}\right) \bar{\beta}_{j}(\gamma, \tau) \\
& +d_{i} \log ^{-1} \tau+\gamma_{i} \log ^{-1} \tau-\left(c L_{i, n}+\bar{P}_{i}(\tau)\right) \log ^{-2} \tau .
\end{aligned}
$$

For each pair of integers $(i, j)$ under consideration, the coefficients $L_{i, j}, H_{i, j}, J_{i, j}, a_{i}, d_{i}$ are real numbers. Moreover $\mathbb{V}_{i}$ is a linear map and

$$
\begin{aligned}
\bar{P}_{i}(\tau) & =\bar{P}_{i, 0}+\tau \log \tau \bar{P}_{i, 1}+\cdots, \\
\bar{R}_{n-1+i}(\tau) & =\bar{R}_{n-1+i, 0}+\tau \log \tau \bar{R}_{n-1+i, 1}+\cdots
\end{aligned}
$$

THEOREM 2. (Dulac case of even codimension) Let

$$
\begin{gathered}
B: \mathbb{R}_{+} \times \mathbb{R}^{n} \times \mathbb{R}^{n} \rightarrow \mathbb{R}, \\
(u, \alpha, \beta) \mapsto B_{\alpha, \beta}(u)=\sum_{i=0}^{n-1} \alpha_{i} u^{i}+\sum_{i=1}^{n} \beta_{i} u^{i} \log u+c\left[u^{n}+\cdots\right],
\end{gathered}
$$

be a non-degenerate Dulac unfolding of codimension $2 n$. Then

$$
B_{\Psi(\gamma, \tau)}(\tau(1+x))=\frac{k(\gamma, \tau) \tau^{n}}{1+\tilde{a}_{n} \log \tau}\left(Q_{2 n}^{s, \pm}(x, \gamma)+\mathcal{O}\left(x^{2 n+1}\right)\right),
$$


where $k(\gamma, \tau) \neq 0$ depends $C^{\infty}$ on $(\gamma, \tau)$, and where

$$
\Psi: \mathbb{R}^{2 n-1} \times \mathbb{R}_{+} \rightarrow \mathbb{R}^{2 n}, \quad(\gamma, \tau) \mapsto(\alpha(\gamma, \tau), \beta(\gamma, \tau)) .
$$

The map $\Psi$ is $C^{\infty}$ and of the form

$$
\begin{gathered}
\alpha_{i}=\tau^{n-i} \bar{\alpha}_{i}(\gamma, \tau), \quad 0 \leq i \leq n-1, \\
\beta_{i}=\frac{\tau^{n-i}}{1+\tilde{a}_{n} \log \tau} \bar{\beta}_{i}(\gamma, \tau), \quad 1 \leq i \leq n .
\end{gathered}
$$

We further specify

$$
\bar{\beta}_{n}(\gamma, \tau)=-c \tilde{a}_{n}+\left(1+\tilde{a}_{n, 1} \log \tau\right)^{-1} \mathbb{W}_{n}(\gamma)-\tau \log \tau Q_{2 n-1}(\tau),
$$

for each integer $i=1, \ldots, n-1$,

$$
\begin{aligned}
\bar{\beta}_{i}(\gamma, \tau)= & -\tilde{a}_{i} \log \tau\left(1+\tilde{a}_{n, 1} \log \tau\right)^{-1} \mathbb{W}_{n}(\gamma)+\mathbb{W}_{i}(\gamma)-c \tilde{a}_{i} \\
& -\tau \log ^{2} \tau \tilde{a}_{n} Q_{n+i-1}(\tau)+\tilde{a}_{i} \tau \log ^{2} \tau Q_{2 n-1}(\tau)-\tau \log \tau Q_{n+i-1}(\tau), \\
\bar{\alpha}_{n-1}(\gamma, \tau)= & -\frac{\log \tau}{1+\tilde{a}_{n} \log \tau} \sum_{j=1}^{n}\left(L_{n-1, j} \log ^{-1} \tau+H_{n-1, j}\right) \bar{\beta}_{j}(\gamma, \tau) \\
& -c L_{n, 0}+\gamma_{n-1}\left(1+\tilde{a}_{n} \log \tau\right)^{-1}+\tau \log \tau R_{n-1}(\tau),
\end{aligned}
$$

and for each integer $i=0, \ldots, n-2$,

$$
\begin{aligned}
\bar{\alpha}_{i}(\gamma, \tau)= & -\sum_{j=i+1}^{n-1} J_{i, j} \bar{\alpha}_{j}(\gamma, \tau)-\frac{\log \tau}{1+\tilde{a}_{n} \log \tau} \sum_{j=1}^{n}\left(L_{i, j} \log ^{-1} \tau+H_{i, j}\right) \bar{\beta}_{j}(\gamma, \tau) \\
& -c L_{i, 0}+\gamma_{i}\left(1+\tilde{a}_{n} \log \tau\right)^{-1}-\tau \log \tau R_{i}(\tau)
\end{aligned}
$$

For each pair of integers $(i, j)$ under consideration, the coefficients $L_{i, j}, H_{i, j}, J_{i, j}, \tilde{a}_{i}$ are real numbers. Moreover, $\mathbb{W}_{i}$ is a linear map and

$$
Q_{i}(\tau)=Q_{i, 0}+\tau \log \tau Q_{i, 1}+\cdots, \quad R_{i}(\tau)=R_{i, 0}+\tau \log \tau R_{i, 1}+\cdots .
$$

1.2.2. The compensator case. In the case $\varepsilon \neq 0$ an asymptotic expansion for $B_{\lambda, \varepsilon}$ in [44] was found to be

$$
\begin{aligned}
B_{\lambda, \varepsilon}(u)= & \alpha_{0}+\beta_{1}\left[u \omega+\varepsilon \eta_{1}(u, u \omega)\right]+\alpha_{1}\left[u+\varepsilon \mu_{1}(u, u \omega)\right]+\cdots \\
& +\alpha_{n}\left[u^{n}+\varepsilon \mu_{n}(u, u \omega)\right]+\beta_{n+1}\left[u^{n+1} \omega+\varepsilon \eta_{n+1}(u, u \omega)\right] \\
& +\Psi_{n}(u, \lambda, \varepsilon),
\end{aligned}
$$

where $\alpha_{i}$ and $\beta_{j+1}$ for $0 \leq j \leq n$ depend $C^{\infty}$ on $(\lambda, \varepsilon)$. Either

$$
\alpha_{i}(0,0)=\beta_{i+1}(0,0)=0, \quad 0 \leq i \leq n-1 \quad \text { and } \quad \alpha_{n}(0,0) \neq 0,
$$

or

$$
\alpha_{i}(0,0)=\beta_{i+1}(0,0)=\alpha_{n}(0,0)=0, \quad 0 \leq i \leq n-2 \quad \text { and } \quad \beta_{n}(0,0) \neq 0 .
$$


Furthermore, $\omega=\omega(u)$ is a compensator function of the form

$$
\omega(u)=\frac{u^{\varepsilon \tilde{\beta}}-1}{\varepsilon \beta} \quad \text { if } \varepsilon \tilde{\beta}=0, \omega(u)=\log u, \text { if } \varepsilon \tilde{\beta}=0,
$$

where $\varepsilon \tilde{\beta}=1-R(P)$ and where $R(P)$ is the absolute value of the ratio of the negative eigenvalue of the linear part of $\mathcal{X}_{\varepsilon, \lambda}$ at the saddle point by the positive one. Moreover, the functions $\eta_{i}$ and $\mu_{i}$ are polynomial in $u$ and $u \omega(u)$, taking the form

$$
\eta_{i}(u, u \omega(u))=\eta_{i, 0} u^{i}+\cdots, \quad \mu_{i}(u, u \omega(u))=\mu_{i, 0} u^{i+1} \omega^{i+1}(u)+\cdots,
$$

where $\eta_{i, 0}$ and $\mu_{i, 0}$ are real numbers. Note that the compensator unfolding deforms the Dulac unfolding and is obtained from the latter by replacing $\log (u)$ by $\omega(u)$. The remainder $\Psi_{n}$ is of class $C^{n}$ and flat of order $n$ at $u=0$. An expansion of the form (19) is said to be a non-degenerate compensator unfolding of codimension $k$ if for $\varepsilon=0$ it is a non-degenerate Dulac unfolding of codimension $k$. Theorems 1 and 2 can be extended for compensator unfoldings as follows.

THEOREM 3. (Compensator case of odd codimension) Let

$$
\begin{gathered}
B: \mathbb{R}_{+} \times \mathbb{R}^{n} \times \mathbb{R}^{n-1} \rightarrow \mathbb{R}, \\
(u, \alpha, \beta) \mapsto B_{\alpha, \beta}(u)=\sum_{i=0}^{n-1} \alpha_{i}\left[u^{i}+\cdots\right]+\sum_{i=1}^{n-1} \beta_{i}\left[u^{i} \omega(u)+\cdots\right]+c\left[u^{n} \omega(u)+\cdots\right],
\end{gathered}
$$

be a non-degenerate compensator unfolding of codimension $2 n-1$. Then

$$
B_{\Psi(\gamma, \tau)}(\tau(1+x))=k(\gamma, \tau, \varepsilon) \tau^{n} \omega(\tau)\left(Q_{2 n-1}^{s, \pm}(x, \gamma)+\mathcal{O}\left(x^{2 n}\right)\right),
$$

where $k(\gamma, \tau, \varepsilon) \neq 0$ depends $C^{\infty}$ on $(\gamma, \tau, \varepsilon)$, and where

$$
\Psi: \mathbb{R}^{2 n-2} \times \mathbb{R}_{+} \rightarrow \mathbb{R}^{2 n-1}, \quad(\gamma, \tau) \mapsto(\alpha(\gamma, \tau), \beta(\gamma, \tau)) .
$$

The map $\Psi$ is $C^{\infty}$ and of the form

$$
\begin{array}{cl}
\alpha_{i}=\tau^{n-i} \omega^{2}(\tau) \bar{\alpha}_{i}(\tau, \gamma, \varepsilon), & 0 \leq i \leq n-1 \\
\beta_{i}=\tau^{n-i} \omega(\tau) \bar{\beta}_{i}(\tau, \gamma, \varepsilon), & 1 \leq i \leq n-1
\end{array}
$$

We further specify

$$
\bar{\beta}_{i}(\tau, \gamma, \varepsilon)=-c \hat{a}_{i}(\tau, \varepsilon)+\mathbb{V}_{n-1+i}(\tau, \gamma, \varepsilon)-\tau \omega(\tau)^{-1} \bar{R}_{n-1+i}(\tau, \varepsilon)
$$

where for each integer $i=n, \ldots, 2 n-2, \hat{V}_{i}$ is linear in $\gamma$ with coefficients depending analytically on $\varepsilon$ and $\tau \omega^{n}(\tau)$. Furthermore

$$
\begin{aligned}
\bar{\alpha}_{n-1}(\tau, \gamma, \varepsilon)= & -\sum_{j=1}^{n-1}\left(\hat{L}_{n-1, j}(\tau, \varepsilon) \omega^{-1}(\tau)+\hat{H}_{n-1, j}(\tau, \varepsilon)\right) \bar{\beta}_{j}(\tau, \gamma, \varepsilon) \\
& +\hat{d}_{n-1}(\tau, \varepsilon) \omega^{-1}(\tau)+\gamma_{n-1} \omega^{-1}(\tau) \\
& -\left(c \hat{L}_{n-1, n}(\tau, \varepsilon)+\bar{P}_{n-1}(\tau, \varepsilon)\right) \omega^{-2}(\tau)
\end{aligned}
$$




$$
\begin{aligned}
\bar{\alpha}_{i}(\tau, \gamma, \varepsilon)= & -\sum_{j=i+1}^{n-1} \hat{J}_{i, j}(\tau, \varepsilon) \bar{\alpha}_{j}(\tau, \gamma, \varepsilon) \\
& -\sum_{j=1}^{n-1}\left(\hat{L}_{i, j}(\tau, \varepsilon) \omega^{-1}(\tau)+\hat{H}_{i, j}(\tau, \varepsilon)\right) \bar{\beta}_{j}(\tau, \gamma, \varepsilon) \\
& +\hat{d}_{i}(\tau, \varepsilon) \omega^{-1}(\tau)+\gamma_{i} \omega^{-1}(\tau)-\left(c \hat{L}_{i, n}(\tau, \varepsilon)+\bar{P}_{i}(\tau)\right) \omega^{-2}(\tau),
\end{aligned}
$$

for $i=0, \ldots, n-2$. For each pair of integers $(i, j)$ under consideration, $\hat{L}_{i, j}, \hat{H}_{i, j}, \hat{J}_{i, j}$, $\hat{a}_{i}, \hat{d}_{i}, \bar{P}_{i}$ and $\bar{R}_{i}$ are analytic functions in $\varepsilon$ and $\tau \omega^{n}(\tau)$.

THEOREM 4. (Compensator case of even codimension) Let

$$
\begin{gathered}
B: \mathbb{R}_{+} \times \mathbb{R}^{n} \times \mathbb{R}^{n} \rightarrow \mathbb{R}, \\
(u, \alpha, \beta) \mapsto B_{\alpha, \beta}(u)=\sum_{i=0}^{n-1} \alpha_{i}\left[u^{i}+\cdots\right]+\sum_{i=1}^{n} \beta_{i}\left[u^{i} \omega(u)+\cdots\right]+c\left[u^{n}+\cdots\right]
\end{gathered}
$$

be a non-degenerate compensator unfolding of codimension $2 n$. Then

$$
B_{\Psi(\gamma, \tau)}(\tau(1+x))=\frac{k(\gamma, \tau, \varepsilon) \tau^{n}}{1+\tilde{a}_{n}(\tau, \varepsilon) \omega(\tau)}\left(Q_{2 n}^{s, \pm}(x, \gamma)+\mathcal{O}\left(x^{2 n+1}\right)\right),
$$

where $k(\gamma, \tau, \varepsilon) \neq 0$ depends $C^{\infty}$ on $(\gamma, \tau, \varepsilon)$, and where

$$
\Psi: \mathbb{R}^{2 n-1} \times \mathbb{R}_{+}, \quad(\gamma, \tau) \mapsto(\alpha(\gamma, \tau), \beta(\gamma, \tau)) .
$$

The map $\Psi$ is $C^{\infty}$ and of the form

$$
\begin{gathered}
\alpha_{i}=\tau^{n-i} \bar{\alpha}_{i}(\tau, \gamma, \varepsilon), \quad 0 \leq i \leq n-1, \\
\beta_{i}=\frac{\tau^{n-i}}{1+\tilde{a}_{n} \omega(\tau)} \bar{\beta}_{i}(\tau, \gamma, \varepsilon), \quad 1 \leq i \leq n
\end{gathered}
$$

We further specify

$$
\bar{\beta}_{n}(\tau, \gamma, \varepsilon)=-c \tilde{a}_{n}(\tau, \varepsilon)+\left(1+\tilde{a}_{n}(\tau, \varepsilon) \omega(\tau)\right)^{-1} \hat{\mathbb{W}}_{n}(\tau, \gamma, \varepsilon)-\tau \omega(\tau) Q_{2 n-1}(\tau, \varepsilon),
$$

for each integer $i=1, \ldots, n-1$,

$$
\begin{aligned}
\bar{\beta}_{i}(\tau, \gamma, \varepsilon)= & -\tilde{a}_{i}(\tau, \varepsilon) \omega(\tau)\left(1+\tilde{a}_{n}(\tau, \varepsilon) \omega(\tau)\right)^{-1} \hat{\mathbb{W}}_{n}(\tau, \gamma, \varepsilon)+\hat{\mathbb{W}}_{i}(\tau, \gamma, \varepsilon)-c \tilde{a}_{i}(\tau, \varepsilon) \\
& -\tau \omega^{2}(\tau) \tilde{a}_{n} Q_{n+i-1}(\tau, \varepsilon)+\tilde{a}_{i}(\tau, \varepsilon) \tau \omega^{2}(\tau) Q_{2 n-1}(\tau, \varepsilon) \\
& -\tau \omega(\tau) Q_{n+i-1}(\tau, \varepsilon)
\end{aligned}
$$

where for each integer $i=1, \ldots, n-1, \mathbb{W}_{i}$ is linear in $\gamma$ with coefficients depending analytically on $\varepsilon$ and $\tau \omega^{n}(\tau)$. Furthermore

$$
\begin{aligned}
\bar{\alpha}_{n-1}(\tau, \gamma, \varepsilon)= & -\frac{\omega(\tau)}{1+\tilde{a}_{n}(\tau, \varepsilon) \omega(\tau)} \sum_{j=1}^{n} \hat{L}_{n-1, j}(\tau, \varepsilon) \omega^{-1}(\tau) \bar{\beta}_{j}(\tau, \gamma, \varepsilon) \\
& -\frac{\omega(\tau)}{1+\tilde{a}_{n}(\tau, \varepsilon) \omega(\tau)} \sum_{j=1}^{n} \hat{H}_{n-1, j}(\tau, \varepsilon) \bar{\beta}_{j}(\tau, \gamma, \varepsilon) \\
& -c \hat{L}_{n, 0}(\tau, \varepsilon)+\gamma_{n-1}\left(1+\tilde{a}_{n}(\tau, \varepsilon) \omega(\tau)\right)^{-1}+\tau \omega(\tau) R_{n-1}(\tau, \varepsilon),
\end{aligned}
$$


and for each $i=1, \ldots, n-2$,

$$
\begin{aligned}
\bar{\alpha}_{i}(\tau, \gamma, \varepsilon)= & -\sum_{j=i+1}^{n-1} \hat{J}_{i, j}(\tau, \varepsilon) \bar{\alpha}_{j}(\tau, \gamma, \varepsilon) \\
& -\frac{\omega(\tau)}{1+\tilde{a}_{n}(\tau, \varepsilon) \omega(\tau)} \sum_{j=1}^{n}\left(\hat{L}_{i, j}(\tau, \varepsilon) \omega^{-1}(\tau)+\hat{H}_{i, j}(\tau, \varepsilon)\right) \bar{\beta}_{j}(\tau, \gamma, \varepsilon) \\
& -c \hat{L}_{i, 0}(\tau, \varepsilon)+\gamma_{i}\left(1+\tilde{a}_{n}(\tau, \varepsilon) \omega(\tau)\right)^{-1}-\tau \omega(\tau) R_{i}(\tau, \varepsilon) .
\end{aligned}
$$

For each pair of integers $(i, j)$ under consideration, $\hat{L}_{i, j}, \hat{H}_{i, j}, \hat{J}_{i, j}, \tilde{a}_{i}, Q_{i}$ and $R_{i}$ are analytic functions in $\varepsilon$ and $\tau \omega^{n}(\tau)$.

Remark. (i) Concerning the topology of the bifurcation set of limit cycles, Marděsic $[34,35]$ shows that the following properties hold. In the Dulac expansion case $(\varepsilon=0)$, the bifurcation diagram is homeomorphic to the bifurcation diagram of the model $Q_{k}^{p, \pm}(u, \alpha)$ in (5); and in the compensator case $(\varepsilon>0)$, the bifurcation diagram is homeomorphic to the product of the interval $\left(0, \varepsilon_{0}\right)$ and the bifurcation diagram of the polynomial model, $Q_{k}^{p, \pm}(u, \beta)$. If we restrict the parameter space to the image of the map $\Psi$ given in Theorems 1-4, these latter properties also directly follow from our approach. Marděsic's approach is rather topological and his results are not suitable for more analytical studies. We emphasize here the explicit character of our results that are preparations for further applications. Indeed, in [13], we apply these results to study quasi-periodic bifurcation of invariant tori for the full system $X_{\lambda, \varepsilon}+\varepsilon^{r} R$. For more details, see $\$ 1.4$ below.

(ii) An interesting observation concerns the image of the scaling and its order of contact with the hyperplane $\left\{\alpha_{0}=0\right\}$ of homoclinic bifurcation. In the case of odd codimension, if a point $(\alpha, \beta)$ belongs to the boundary of the image of the scaling, from (16) it follows that for all $r>0$ there exists a constant $\mathrm{C}>1$ such that, for each integer $i=1, \ldots, n-1$,

$$
\mathrm{C}\left|\beta_{n-1}\right|^{n-i} \leq \max \left\{\left|\alpha_{i}\right|,\left|\beta_{i}\right|\right\} \leq \mathrm{C}\left|\beta_{n-1}\right|^{n-i-r}
$$

and

$$
\mathrm{C}\left|\beta_{n-1}\right|^{n} \leq\left|\alpha_{0}\right| \leq \mathrm{C}\left|\beta_{n-1}\right|^{n-r} .
$$

This implies that the contact is $n$-flat; compare with Example 2. However, in the case of even codimension, from (18) it follows that for all $r>0$ there exists a constant $\tilde{C}>1$ such that

$$
\begin{aligned}
& \left|\beta_{i}\right| \leq \tilde{C} \exp \left(-\frac{n-i}{\left|\beta_{n-1}\right|}\right), \quad i=1, \ldots, n-2, \\
& \left|\alpha_{i}\right| \leq \tilde{C} \exp \left(-\frac{n}{\left|\beta_{n-1}\right|}\right), \quad i=0, \ldots, n-1 .
\end{aligned}
$$

This implies that the contact is infinitely flat and that the bifurcation set is located in an exponentially narrow horn; compare with $\S 1.3$. Furthermore, the region in the phase space under consideration is an exponentially thin annulus. This observation reveals a significant difference between the odd- and the even-codimension cases.

(iii) Concerning the geometry of the bifurcation set of limit cycles, from formulae (15) and (17) we clearly obtain the hierarchy of the standard catastrophe theory in terms of the 
parameter $\gamma$ and this hierarchy is independent of $\tau$. In this sense we can say that, along the curve $\mathcal{C}$, the singularity has been removed. From this, using formulae (16) and (18), we can deduce a hierarchy for the bifurcation sets in the parameters $(\alpha, \beta)$ which now depends on $\tau$ in a non-regular manner when $\tau$ approaches 0 . It would be of interest, at least in the odd-codimension case, to further study the geometry of the bifurcation set of limit cycles, for instance the order of contact between the different subordinate $d$-fold bifurcations in a family of cuspoids of degree $k>d$. However, when working in the range of the scaling, it is appropriate to work in terms of the parameter $\gamma$ and the variable $x$.

1.3. Application: the codimension three saddle connection. Consider the following unfolding of the codimension four Bogdanov-Takens bifurcation

$$
\begin{gathered}
\dot{x}=y, \\
\dot{y}=x^{2}+\mu+y\left(v_{0}+v_{1} x+v_{2} x^{2} \pm x^{4}\right) .
\end{gathered}
$$

This family was partially studied in $[\mathbf{2 3}, \mathbf{3 1}]$ for more background see $[\mathbf{2 4}, \mathbf{2 5}, \mathbf{2 8}, \mathbf{2 9}, \mathbf{5 0}]$. After the rescaling

$$
\mu=-\varepsilon^{4}, \quad \nu_{0}=\varepsilon^{8} \lambda_{0}, \quad \nu_{1}=\varepsilon^{6} \lambda_{1}, \quad \nu_{2}=\varepsilon^{4} \lambda_{2}, \quad x=\varepsilon^{2} \bar{x}, \quad y=\varepsilon^{3} \bar{y},
$$

and then omitting all bars, we end up with the following three-parameter family of vector fields:

$$
\begin{gathered}
\dot{x}=\varepsilon y, \\
\dot{y}=\varepsilon\left(\left(x^{2}-1\right)+\varepsilon^{5} y\left(\lambda_{0}+\lambda_{1} x+\lambda_{2} x^{3} \pm x^{4}\right)+\mathcal{O}\left(\varepsilon^{r}\right)\right) .
\end{gathered}
$$

In terms of our general system (1) we have $p=1$ and $q=8, r>8$, i.e.

$$
\mathcal{X}_{\lambda, \varepsilon}=\varepsilon X_{H}+\varepsilon^{8} Y_{\lambda, \varepsilon}
$$

where

$$
H(\bar{x}, \bar{y})=\frac{1}{2} \bar{y}^{2}-\frac{1}{3} \bar{x}^{3}+\bar{x} .
$$

The scaled displacement function takes the form

$$
B_{\lambda, \varepsilon}(u)=\alpha_{0}+\beta_{1}[u \omega(u)+\cdots]+\alpha_{1}[u+\cdots]+c\left[u^{2} \omega(u)+\cdots\right]+\mathcal{O}\left(u^{3}\right) .
$$

For simplicity, we again restrict to the case $\varepsilon=0$. The scaling

$$
\Psi(\gamma, \tau)=(\alpha(\gamma, \tau), \beta(\gamma, \tau))
$$

of Theorem 1 takes the explicit form

$$
\begin{gathered}
\beta_{1}=-3 c \tau-2 c \tau \log \tau+\tau^{2} \bar{R}_{2}(\tau), \\
\alpha_{0}=c \tau^{2} \log \tau+\gamma_{0} \tau^{2} \log \tau+\gamma_{1} \tau^{2} \log \tau+\tau^{2} \bar{P}_{0}(\tau), \\
\alpha_{1}=3 c \tau \log \tau+2 c \tau \log ^{2} \tau+\gamma_{1} \tau \log \tau+\tau \bar{P}_{1}(\tau) .
\end{gathered}
$$

Therefore

$$
\begin{aligned}
B_{\Psi(\gamma, \tau), 0}(\tau(1+x)) & =\tau^{2} \log \tau U(x, \gamma, \tau)\left(\gamma_{0}+\gamma_{1} x \pm x^{3}\right) \\
& =\tilde{U}(x, \gamma, \tau) Q_{3}^{s, \pm}(x, \gamma),
\end{aligned}
$$


where $U$ is given by the division theorem. The latter function, although smooth if $\tau>0$, has Dulac asymptotics at $\tau=0$. Up to a division by $\tilde{U}$, the scaled displacement function is the normal form of a cusp. The bifurcation set of limit cycles $\mathcal{B}$ is given by $\mathcal{B}=\Psi(\mathcal{V})$ where

$$
\mathcal{V}=\left\{\left(\tau,-3 x^{2}, 2 x^{3}\right) \mid \tau>0, x \in \mathbb{R}\right\},
$$

which is the product of a cusp and an interval. From (22) we observe that if $\left(\alpha_{0}, \beta_{1}, \alpha_{1}\right)$ belongs to the bifurcation set of limit cycles, the estimates

$$
\left|\alpha_{1}\right| \leq\left|\beta_{1}\right| \leq\left|\alpha_{1}\right|^{1-r}, \quad\left|\alpha_{1}\right|^{2} \leq\left|\alpha_{0}\right| \leq \frac{1}{c}\left|\alpha_{1}\right|^{2-r}
$$

hold for any $r>0$. This implies that, contrary to the even-codimension case (see Example 2), the order of contact between the bifurcation set of limit cycles and the hyperplane $\left\{\alpha_{0}=0\right\}$ of homoclinic bifurcation is finite.

1.4. Further applications. We summarize as follows. We are given a family of vector fields of the form (1),

$$
X_{\lambda, \varepsilon}(x, y)+\varepsilon^{r} R(x, y, t, \lambda, \varepsilon),
$$

with $x, y, t \in \mathbb{R}, \lambda \in \mathbb{R}^{\ell}$ and where $\varepsilon \geq 0$ is a real perturbation parameter. The autonomous part $X_{\lambda, \varepsilon}=\varepsilon^{p} X_{H}+\varepsilon^{q} Y_{\lambda, \varepsilon}$ is a such that $X_{H}$ is a Hamiltonian with Hamilton function $H$, which is of Morse type, and $Y_{\lambda, \varepsilon}$ is a dissipative family. Moreover, $X_{H}$ possesses a homoclinic loop $\Gamma$. The dissipative part is generic in an appropriate sense. In this setting we focus our study near the homoclinic loop $\Gamma$ and our concern is with the autonomous family $X_{\lambda, \varepsilon}$. By an explicit scaling, a narrow fine structure for the bifurcation set of limit cycles is found in the parameter space $\mathbb{R}^{\ell}=\{\lambda\}$. We show that the corresponding geometry is polynomial where a scaled displacement function can display the full complexity of the cuspoid family

$$
Q_{k}^{s, \pm}(x, \gamma)=\gamma_{0}+\gamma_{1} x+\cdots+\gamma_{k-2} x^{k-2} \pm x^{k}
$$

where $\gamma=\gamma(\lambda)$ is appropriate; compare with (4).

We now discuss certain consequences of the above results for the full non-autonomous family $X_{\lambda, \varepsilon}+\varepsilon^{r} R$, see (1), where $R$ is assumed time-periodic of period $2 \pi$ and therefore has the solid torus $\mathbb{R}^{2} \times \mathbb{S}^{1}$ as its phase space. Here the limit cycles of the 'unperturbed' part $X_{\lambda, \varepsilon}$ correspond to invariant 2-tori with parallel dynamics $[12,11]$. By normal hyperbolicity these tori persist as long as the multiparameter $\lambda$ remains outside an exponentially narrow neighbourhood of the bifurcation set of limit cycles found before; compare with [14] for details.

Near the bifurcation set of limit cycles we can apply quasi-periodic bifurcation theory as developed by $[\mathbf{2}, \mathbf{8 - 1 0}, \mathbf{1 2}, \mathbf{1 3}, \mathbf{2 0}, \mathbf{3 9}, \mathbf{5 2}, \mathbf{5 3}]$. To this end we consider 'unperturbed' tori which are Diophantine. This means that the two frequencies $(1, \omega(\lambda))$ are such that

$$
\left|\omega(\lambda)-\frac{p}{q}\right| \geq \kappa q^{-v}
$$

for certain $\kappa>0$ and $v>2$. For fixed $\kappa$ and $v$, formula (24) inside $\mathbb{R}^{\ell}=\{\lambda\}$ defines a Cantor foliation of hypersurfaces. At the intersection of this foliation with the 
bifurcation set, the 'unperturbed' family $X_{\lambda, \varepsilon}$ displays a cuspoid bifurcation pattern of 2-tori as can be directly inferred from the above. In the 'perturbed' case $X_{\lambda, \varepsilon}+\varepsilon^{r} R$, we conjecture persistence of many of these Diophantine tori, including the bifurcation pattern, provided that $|\varepsilon| \ll 1$. Here $\kappa=\kappa(\varepsilon)=o(\varepsilon)$ has to be chosen appropriately. For instance, whenever the Cantor foliation intersects a fold hyperplane transversally, we expect a quasi-periodic saddle node bifurcation of 2-tori to occur $[\mathbf{1 1}, \mathbf{1 2}]$. These KAM persistence results involve a Whitney smooth reparametrization which is near the identity map in terms of $|\varepsilon| \ll 1$. This means that the perturbed Diophantine 2-tori correspond to a perturbed Cantor foliation in the parameter space $\mathbb{R}^{\ell}=\{\lambda\}$. In the complement of this perturbed Cantor foliation of hypersurfaces we expect all the dynamical complexity regarding Cantori, strange attractors, etc., as described in [19-22, 38, 43]. This programme will be the subject of $[\mathbf{1 3}]$ where we aim to apply $[\mathbf{1 0}, \mathbf{1 1}, \mathbf{1 2}, \mathbf{5 1}, \mathbf{5 2}]$ to establish the occurrence of quasi-periodic cuspoid bifurcations in the three cases (a), (b) and (c) of Figure 1.

A second topic is the generalization of the approach of the present paper to cases where the Hamiltonian $H$ in (1) no longer is a (stable) Morse function, but undergoes simple bifurcations.

\section{Proofs}

We first give a proof of Theorems 1 and 2. Theorems 3 and 4 generalize Theorems 1 and 2 to compensator unfoldings and are obtained by a perturbation method. Before we distinguish between the odd- and even-codimension cases, we first describe the main idea. Consider a non-degenerate Dulac unfolding of the form

$$
B_{\alpha, \beta}(u)=\sum_{j=0}^{n} \alpha_{j} u^{j}+\sum_{j=1}^{n} \beta_{j} u^{j} \log u+\tilde{H}(u),
$$

where $\tilde{H}(u)=c u^{n+1} \log u+\cdots$. Put

$$
u=\tau(1+x) \quad \text { and } \quad B_{\alpha, \beta}(\tau(1+x))=\Phi_{\alpha, \beta}(x) .
$$

Using the Taylor formula we get

$$
\Phi_{\alpha, \beta}(x)=\sum_{i=0}^{2 n} \Lambda_{i} x^{i}+\mathcal{O}\left(x^{2 n+1}\right) .
$$

Since the Dulac unfolding is non-degenerate we can see the coefficients of the unfolding as independent. The goal of this section is to show that independent coefficients of the Dulac unfolding yield independent coefficients for the monomials $x^{i}$ in (26). It will also be proven that the non-zero leading term in (25) ( $\alpha_{n}$ for even and $\beta_{n}$ for odd codimension) leads to a non-zero leading term in (26). To illustrate this, we consider the case of codimension $k=2 n-1$, i.e. where $\beta_{n} \neq 0$. This case is covered by Theorem 1 and in (11) we have $\tilde{f}(\tau)=\tau^{n} \log \tau$. It follows that indeed the leading term in (26) is of degree $2 n-1$; compare with Example 2 for a special case. In the case of codimension $k=2 n$ covered by Theorem 2 , we similarly have $\tilde{f}(\tau)=\tau^{n} /(1+\log \tau)$ in $(11)$; compare with $\S 1.3$ for a special case. 
To be precise, for each integer $i=0, \ldots, 2 n$, in (26) we have

$$
\Lambda_{i}=\frac{\Phi^{(i)}(0)}{i !}=\frac{\tau^{i}}{i !} B_{\alpha, \beta}^{(i)}(\tau)
$$

and together with (25) we get

$$
\Lambda_{i}=\frac{\tau^{i}}{i !} \sum_{j=0}^{n} \alpha_{j}\left(u^{j}\right)_{u=\tau}^{(i)}+\frac{\tau^{i}}{i !} \sum_{j=1}^{n} \beta_{j}\left(u^{j} \log u\right)_{u=\tau}^{(i)}+\frac{\tau^{i}}{i !} \tilde{H}^{(i)}(\tau) .
$$

Observe that the following equalities hold:

$$
\begin{gathered}
\frac{\tau^{i}}{i !} \alpha_{j}\left(u^{j}\right)_{u=\tau}^{(i)}=\alpha_{j} \tau^{j}\left(\frac{j !}{i !(j-i) !}\right), \quad \text { if } j \geq i, \\
\frac{\tau^{i}}{i !} \alpha_{j}\left(u^{j}\right)_{u=\tau}^{(i)}=0, \quad \text { if } j<i, \\
\frac{\tau^{i}}{i !} \beta_{j}\left(u^{j} \log u\right)_{u=\tau}^{(i)}=(-1)^{i-j-1} \frac{j !(i-j-1) !}{i !} \beta_{j} \tau^{j}, \quad \text { if } j<i
\end{gathered}
$$

and

$$
\frac{\tau^{i}}{i !} \tilde{H}^{(i)}(\tau)=\tau^{n+1} \log \tau R_{i}(\tau)
$$

where

$$
R_{i}(\tau)=R_{i, 0}+R_{i, 1} \tau \log \tau+\cdots .
$$

We use the Leibniz rule to compute $\left(u^{j} \log u\right)_{u=\tau}^{(i)}$, when $i \leq j$, and we get

$$
\begin{aligned}
\frac{\tau^{i}}{i !} \beta_{j}\left(u^{j} \log u\right)_{u=\tau}^{(i)} & =\beta_{j} \frac{\tau^{i}}{i^{!}} \sum_{s=0}^{i}\left(\begin{array}{l}
i \\
s
\end{array}\right)\left(u^{j}\right)_{u=\tau}^{(i-s)}(\log u)_{u=\tau}^{(s)} \\
& =\beta_{j} \frac{\tau^{j}}{i !} \log \tau \frac{j !}{(j-i) !}+\beta_{j} \frac{\tau^{j}}{i !} \sum_{s=1}^{i}\left(\begin{array}{l}
i \\
s
\end{array}\right)\left(u^{j}\right)_{u=\tau}^{(i-s)}(\log u)_{u=\tau}^{(s)} \\
& =\beta_{j} \frac{\tau^{j}}{i !} \log \tau \frac{j !}{(j-i) !}+\beta_{j} \frac{\tau^{j}}{i !} \sum_{s=1}^{i}\left(\begin{array}{l}
i \\
s
\end{array}\right) \frac{(-1)^{s-1}(s-1) ! j !}{(j-i+s) !}
\end{aligned}
$$

Observe that

$$
\frac{\tau^{i}}{i !}\left(u^{j} \log u\right)_{u=\tau}^{(i)}=\frac{\tau^{j}}{i !} \log \tau \frac{j !}{(j-i) !}+\frac{\tau^{j}}{i !}\left(u^{j} \log u\right)_{u=1}^{(i)},
$$

where

$$
\begin{aligned}
\frac{1}{i !}\left(u^{j} \log u\right)_{u=1}^{(i)} & =\sum_{s=1}^{i}\left(\begin{array}{l}
i \\
s
\end{array}\right) \frac{(-1)^{s-1}(s-1) ! j !}{i !(j-i+s) !} \\
& =\sum_{s=1}^{i} \frac{(-1)^{s-1} j !}{(j+s-i) !(i-s) ! s} .
\end{aligned}
$$

We then introduce the following notation. Let

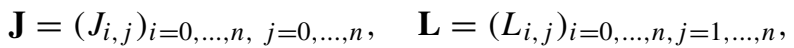

$$
\begin{aligned}
& \mathbf{H}=\left(H_{i, j}\right)_{i=0, \ldots, n, j=1, \ldots, n} \quad \text { and } \quad \mathbf{T}=\left(T_{i, j}\right)_{i=n+1, \ldots, 2 n, j=1, \ldots, n},
\end{aligned}
$$


be respectively the $(n+1) \times(n+1),(n+1) \times n,(n+1) \times n$ and $n \times n$ matrices defined as follows:

$$
\begin{gathered}
J_{i, j}=H_{i, j}=\left(\begin{array}{l}
j \\
i
\end{array}\right) \quad \text { if } i \leq j, \\
J_{i, j}=H_{i, j}=0 \quad \text { if } j<i, \\
L_{i, j}=\frac{(-1)^{i-j-1}}{i !} j !(i-j-1) ! \quad \text { if } j<i, \\
L_{i, j}=\frac{1}{i !}\left(u^{j} \log u\right)_{u=1}^{(i)}=\sum_{s=1}^{i} \frac{(-1)^{s-1} j !}{(j+s-i) !(i-s) ! s} \quad \text { if } i \leq j,
\end{gathered}
$$

and

$$
T_{i, j}=\frac{(-1)^{i-j+1}}{i !} j !(i-j-1) !
$$

Finally $\tilde{\mathbf{N}}=\left(\tilde{N}_{i, j}\right)_{i=n, \ldots, 2 n-1, j=1, \ldots, n}$ is defined as follows:

$$
\tilde{N}_{n, n}=1, \quad \tilde{N}_{i, j}=0 \quad \text { if }(i, j) \neq(n, n) .
$$

We also introduce the following matrices:

$$
\begin{aligned}
& \mathbf{J}^{-}=\left(J_{i, j}^{-}\right)_{i=0, \ldots, n-1, j=0, \ldots, n-1,} \quad \text { where } J_{i, j}^{-}=J_{i, j}, \\
& \mathbf{H}^{-}=\left(H_{i, j}^{-}\right)_{i=0, \ldots, n-1, j=1, \ldots, n,} \text { where } H_{i, j}^{-}=H_{i, j},
\end{aligned}
$$

and

$$
\mathbf{L}^{-}=\left(L_{i, j}^{-}\right)_{i=0, \ldots, n-1, j=1, \ldots, n}, \quad \text { where } L_{i, j}^{-}=L_{i, j} .
$$

With the above notation we get

$$
\Lambda_{i}=\sum_{j=0}^{n} J_{i, j} \alpha_{j} \tau^{j}+\sum_{j=1}^{n}\left(H_{i, j} \log \tau+L_{i, j}\right) \beta_{j} \tau^{j}+\tau^{n+1} \log \tau R_{i}(\tau),
$$

if $i \leq n$, and

$$
\begin{aligned}
\Lambda_{i} & =\sum_{j=1}^{n} \beta_{j} \tau^{j} \frac{(-1)^{i-j-1}}{i !} j !(i-j-1) !+\tau^{n+1} \log \tau R_{i}(\tau) \\
& =\sum_{j=1}^{n} T_{i, j} \beta_{j} \tau^{j}+\tau^{n+1} \log \tau R_{i}(\tau),
\end{aligned}
$$

if $i>n$. We state the following lemma.

LEMMA 1. Let

$$
\begin{gathered}
\tilde{\mathbf{A}}=\left(\tilde{A}_{i, j}\right)_{i=n, \ldots, 2 n-1, j=1, \ldots, n}, \quad \tilde{A}_{i, j}=T_{i, j} \text { if } i>n, \quad \tilde{A}_{n, j}=L_{n, j}, \\
\mathbf{A}=\left(A_{i, j}\right)_{i=n, \ldots, 2 n-2, j=1, \ldots, n-1}, \quad A_{i, j}=\tilde{A}_{i, j}, \\
\mathbf{B}=\left(B_{i, j}\right)_{i=n+1, \ldots, 2 n-1, j=1, \ldots, n-1}, \quad B_{i, j}=A_{i, j} .
\end{gathered}
$$

Then $\mathbf{A}, \mathbf{B}, \tilde{\mathbf{A}}$ and $\mathbf{T}$ are invertible. 
Proof. We show that $\tilde{\mathbf{A}}$ is invertible. The non-degeneracy of the three other matrices follows exactly the same argument. First of all, we claim that $L_{i, j}>0$, for each integer $1 \leq i \leq j \leq n$. To show this, recall that

$$
L_{i, j}=\frac{1}{i !}\left(u^{j} \log u\right)_{u=1}^{(i)} .
$$

It then follows that

$$
\begin{aligned}
L_{i+1, j+1} & =\frac{1}{(i+1) !}\left((j+1) u^{j} \log u+u^{j}\right)_{u=1}^{(i)} \\
& =\frac{j+1}{i+1} L_{i, j}+\frac{j !}{(i+1) !(j-i) !} .
\end{aligned}
$$

In particular, for each integer $1 \leq i \leq n$,

$$
L_{i, i}=\sum_{k=1}^{i} \frac{1}{i}
$$

Since for each integer $1 \leq s \leq n$,

$$
L_{1, s}=\left(u^{s} \log u\right)_{u=1}^{\prime}=1,
$$

it turns out that for each integer $s=1, \ldots, n, L_{1, s}$ is always positive. With (30) it then follows that for each integer $1 \leq i \leq j \leq n$, the $L_{i, j}$ are always positive.

By definition

$$
\left(\tilde{A}_{i, j}\right)_{i=n, \ldots, 2 n-1, j=1, \ldots, n}=\frac{1}{i !}\left(\left(u^{j} \log u\right)_{u=1}^{(i)}\right)_{i=n, \ldots, 2 n-1, j=1, \ldots, n} .
$$

Thus

$$
\operatorname{det}\left(\tilde{A}_{i, j}\right)=\frac{1}{\prod_{i=n}^{2 n-1} i !} \operatorname{det}\left(\left(u^{j} \log u\right)_{u=1}^{(i)}\right) .
$$

For each integer $j=1, \ldots, n$, we put $f_{j}(u)=\left(u^{j} \log u\right)^{(n)}$. Then

$$
\operatorname{det}\left(\tilde{A}_{i, j}\right)=\frac{1}{\prod_{i=n}^{2 n-2} i !} W\left(f_{1}, \ldots, f_{n}\right)(1),
$$

where

$$
W\left(f_{1}, \ldots, f_{n}\right)(u)=\left|\begin{array}{ccc}
f_{1}(u) & \ldots & f_{n}(u) \\
f_{1}^{\prime}(u) & \ldots & f_{n}^{\prime}(u) \\
\vdots & \ddots & \vdots \\
f_{1}^{n-1}(u) & \ldots & f_{n-1}^{n-1}(u)
\end{array}\right| .
$$

Let $g: \mathbb{R} \rightarrow \mathbb{R}, u \mapsto g(u)$ be a $C^{\infty}$ function. We have that

$$
W\left(g f_{1}, \ldots, g f_{n}\right)(u)=g^{n} W\left(f_{1}, \ldots, f_{n}\right)(u) .
$$

This latter property can be shown by induction on $n$ using the fact that the determinant is multilinear. Take

$$
g(u)=\left(-1^{n}\right) u^{n-1} /(n-1) !
$$


We then get

$$
W\left(g f_{1}, \ldots, g f_{n}\right)(u)=\left|\begin{array}{ccccc}
1 & k_{1} u & k_{2} u^{2} & \ldots & k_{n-1} u^{n-1}+(-1)^{n} n \log u \\
0 & k_{1} & 2 k_{2} u & \ldots & (n-1) k_{n-1} u^{n-2} \\
0 & 0 & 2 k_{2} & \ldots & (n-1)(n-2) k_{n-1} u^{n-3} \\
\vdots & \vdots & \vdots & \ddots & \vdots \\
0 & 0 & 0 & \ldots & k_{n-1}(n-1) !
\end{array}\right|
$$

where for each integer $j=1, \ldots, n-1$,

$$
k_{j}=\frac{(-1)^{n}}{(n-1) !}\left(u^{j} \log u\right)_{u=1}^{n}=\frac{(-1)^{n} j !}{(n-1) !} L_{n, j} \neq 0 .
$$

This implies that

$$
W\left(g f_{1}, \ldots, g f_{n}\right)(1)=\prod_{j=1}^{n-1} j ! k_{j} \neq 0
$$

which ends the proof of Lemma 1.

From now on we need to distinguish between the odd-codimension case (developed in $\$ 2.1$ ) and the even-codimension case (developed in $\$ 2.2$ ).

2.1. Proof of Theorem 1. In the odd case $k=2 n-1$, the parameters are $\alpha_{0}, \ldots, \alpha_{n-1}, \beta_{1}, \ldots, \beta_{n-1}$. Moreover we have $\beta_{n}=c \neq 0$ is a constant. This implies that

$$
B_{\alpha, \beta}(u)=\sum_{i=0}^{n-1} \alpha_{i} u^{i}+\sum_{i=1}^{n-1} \beta_{i} u^{i} \log u+c u^{n} \log u+u^{n} \mathcal{F}(u),
$$

where

$$
\mathcal{F}(u)=\mathcal{F}_{0}+\mathcal{F}_{1} u \log u+\cdots
$$

In this situation, the term $\alpha_{n} u^{n}$ in (25) is included in the term $u^{n} \mathcal{F}(u)$. By putting $x=\tau(1+x)$ and $B_{\alpha, \beta}(\tau(1+x))=\Phi_{\alpha, \beta}(x)$, we get

$$
\Phi_{\alpha, \beta}(x)=\Lambda_{0}+\Lambda_{1} x+\cdots+\Lambda_{2 n-2} x^{2 n-2}+\Lambda_{2 n-1} x^{2 n-1}+\mathcal{O}\left(x^{2 n}\right) .
$$

With (27), (28) and (29) the following equalities hold:

$$
\begin{aligned}
\left(\begin{array}{c}
\Lambda_{0} \\
\vdots \\
\Lambda_{n-1}
\end{array}\right)= & \mathbf{J}^{-}\left(\begin{array}{c}
\alpha_{0} \\
\alpha_{1} \tau \\
\vdots \\
\alpha_{n-1} \tau^{n-1}
\end{array}\right)+\log \tau \mathbf{H}^{-}\left(\begin{array}{c}
\beta_{1} \tau \\
\vdots \\
\beta_{n-1} \tau^{n-1} \\
c \tau^{n}
\end{array}\right) \\
& +\mathbf{L}^{-}\left(\begin{array}{c}
\beta_{1} \tau \\
\vdots \\
\beta_{n-1} \tau^{n-1} \\
c \tau^{n}
\end{array}\right)+\left(\begin{array}{c}
P_{0}(\tau) \\
\vdots \\
P_{n-2}(\tau) \\
P_{n-1}(\tau)
\end{array}\right)
\end{aligned}
$$




$$
\left(\begin{array}{c}
\Lambda_{n} \\
\vdots \\
\Lambda_{2 n-2} \\
\Lambda_{2 n-1}
\end{array}\right)=(\tilde{\mathbf{A}}+\log \tau \tilde{\mathbf{N}})\left(\begin{array}{c}
\beta_{1} \tau \\
\vdots \\
\beta_{n-1} \tau^{n-1} \\
c \tau^{n}
\end{array}\right)+\left(\begin{array}{c}
P_{n}(\tau) \\
\vdots \\
P_{2 n-2}(\tau) \\
P_{2 n-1}(\tau)
\end{array}\right)
$$

where, for each integer $i \in\{n, n+1, \ldots, 2 n-1\}, P_{i}(\tau)$ takes the form

$$
P_{i}(\tau)=\tau^{n} \bar{P}_{i}(\tau)=\tau^{n}\left(\bar{P}_{i, 0}+\tau \log \tau \bar{P}_{i, 0}+\cdots\right) .
$$

We have the following strategy. We aim to find a scaled reparametrization of the form

$$
\Psi(\gamma, \tau)=(\alpha, \beta)
$$

linking $\gamma=\left(\gamma_{0}, \ldots, \gamma_{2 n-3}\right)$ and $\tau$ with $(\alpha, \beta)$. For each integer $i=1, \ldots, n-1$, we require $\Psi$ to have the components

$$
\alpha_{i}=\tau^{n-i} \log ^{2} \tau \bar{\alpha}_{i}(\gamma, \tau), \quad \beta_{i}=\tau^{n-i} \log \tau \bar{\beta}_{i}(\gamma, \tau),
$$

leading to

$$
\Phi_{\Psi(\gamma, \tau)}(x)=\tilde{f}(\tau)\left(\gamma_{0}+\gamma_{1} x+\cdots+\gamma_{2 n-3} x^{2 n-3}+k(\gamma, \tau) x^{2 n-1}+\mathcal{O}\left(x^{2 n}\right)\right) .
$$

We first determine $\tilde{f}(\tau)$ and show that the leading term $k(\gamma, \tau)$ is such that $k(0,0) \neq 0$. Note that the set $\Psi(0, \tau)$ parametrizes the curve $\mathcal{C}$ of 'highest degeneracy' of $\Phi_{\Psi(\gamma, \tau)}$. We then obtain the desired expression for $\Phi_{\Psi(\gamma, \tau)}$ by solving

$$
\Lambda_{i}=\tilde{f}(\tau) \gamma_{i}, \quad i=1, \ldots, 2 n-2
$$

To be more precise, we start by solving the equation

$$
\Lambda_{i}=0, \quad i=n, \ldots, 2 n-2 .
$$

With (33) and the definition of the matrix $\mathbf{A}$, we get

$$
\begin{aligned}
0 & =\left(\begin{array}{c}
\Lambda_{n} \\
\vdots \\
\Lambda_{2 n-2}
\end{array}\right) \\
& =\mathbf{A}\left(\begin{array}{c}
\beta_{1} \tau \\
\vdots \\
\beta_{n-1} \tau^{n-1}
\end{array}\right)+c \tau^{n} \log \tau\left(\begin{array}{c}
1 \\
0 \\
\vdots \\
0
\end{array}\right)+c \tau^{n}\left(\begin{array}{c}
\tilde{A}_{n, n} \\
\vdots \\
\tilde{A}_{2 n-2, n}
\end{array}\right)+\left(\begin{array}{c}
P_{n}(\tau) \\
\vdots \\
P_{2 n-2}(\tau)
\end{array}\right) .
\end{aligned}
$$

By Lemma 1, the matrix $\mathbf{A}$ is invertible. Let

$$
\mathbf{A}^{-1}=\left(a_{i, j}\right)_{i=1, \ldots, n-1, j=1, \ldots, n-1}
$$

be its inverse. After multiplication by $\mathbf{A}^{-1}$ on the left-hand side and the right-hand side of (35) we get

$$
\left(\begin{array}{c}
\beta_{1} \tau \\
\vdots \\
\beta_{n-1} \tau^{n}
\end{array}\right)=-c \tau^{n} \log \tau\left(\begin{array}{c}
a_{1,1} \\
\vdots \\
a_{n-1,1}
\end{array}\right)+\tau^{n}\left(\begin{array}{c}
\bar{R}_{n}(\tau) \\
\vdots \\
\bar{R}_{2 n-2}(\tau)
\end{array}\right)
$$


where

$$
\left(\begin{array}{c}
\bar{R}_{n}(\tau) \\
\vdots \\
\bar{R}_{2 n-2}(\tau)
\end{array}\right)=-c \mathbf{A}^{-1}\left(\begin{array}{c}
\tilde{A}_{n, n} \\
\vdots \\
\tilde{A}_{2 n-2, n}
\end{array}\right)-\mathbf{A}^{-1}\left(\begin{array}{c}
\bar{P}_{n}(\tau) \\
\vdots \\
\bar{P}_{2 n-2}(\tau)
\end{array}\right)
$$

Observe that, for each integer $i=1, \ldots, n-2$,

$$
\bar{R}_{i}(\tau)=\bar{R}_{i, 0}+\tau \log \tau \bar{R}_{i, 0}+\cdots .
$$

We now set $\gamma=0$ in (34). With (35) and the definition of the matrix $\mathbf{B}$ we have

$$
\begin{aligned}
\left(\begin{array}{c}
\Lambda_{n+1}(0, \tau) \\
\vdots \\
\Lambda_{2 n-1}(0, \tau)
\end{array}\right) & =\mathbf{B}\left(\begin{array}{c}
\beta_{1} \tau \\
\vdots \\
\beta_{n-1} \tau^{n-1}
\end{array}\right)+c \tau^{n}\left(\begin{array}{c}
\tilde{A}_{n+1, n} \\
\vdots \\
\tilde{A}_{2 n-1, n}
\end{array}\right)+\left(\begin{array}{c}
P_{n+1}(\tau) \\
\vdots \\
P_{2 n-1}(\tau)
\end{array}\right) \\
& =\tau^{n} \log \tau \mathbf{B}\left(\begin{array}{c}
\bar{\beta}_{1}(0, \tau) \\
\vdots \\
\bar{\beta}_{n-1}(0, \tau)
\end{array}\right)+c \tau^{n}\left(\begin{array}{c}
\tilde{A}_{n+1, n}+\bar{P}_{n+1}(\tau) \\
\vdots \\
\tilde{A}_{2 n-1, n}+\bar{P}_{n-1}(\tau)
\end{array}\right) .
\end{aligned}
$$

Since $\mathbf{A}^{-1}$ is invertible we have $\left(a_{1,1}, \ldots, a_{n-1,1}\right) \neq 0$. Therefore after division by $\tau^{n} \log \tau$ on both sides of (36), we get

$$
\left(\bar{\beta}_{1}(0, \tau), \ldots, \bar{\beta}_{n-1}(0, \tau)\right) \neq(0, \ldots, 0) .
$$

Moreover, since $\mathbf{B}$ is non-degenerate, with (38) we must have

$$
\sup _{n+1 \leq i \leq 2 n-1}\left|\Lambda_{i}(0, \tau)\right|=-\tau^{n}|\tilde{k}(\tau)| \log \tau,
$$

where $\tilde{k}(0) \neq 0$. But

$$
\left(\Lambda_{n+1}(0, \tau), \ldots, \Lambda_{2 n-2}(0, \tau)\right)=(0, \ldots, 0)
$$

which implies that

$$
\Lambda_{2 n-1}(0, \tau)=\tilde{k}(\tau) \tau^{n} \log \tau,
$$

with

$$
\tilde{k}(\tau)=\sum_{j=1}^{n-1} B_{n-1, j} \bar{\beta}_{j}(0, \tau)+c \log ^{-1} \tau\left(\tilde{A}_{2 n-1, n}+P_{n-1}(\tau)\right) .
$$

As we announced before, we set $\tilde{f}(\tau)=\tau^{n} \log \tau$. We now develop the scaling. For each integer $i=0, \ldots, 2 n-2$, we put

$$
\Lambda_{i}(\gamma, \tau)=\gamma_{i} \tau^{n} \log \tau, \quad \text { for } i \leq 2 n-3, \quad \Lambda_{2 n-2}(\gamma, \tau) \equiv 0 .
$$

From (35) and (37) we then get

$$
\mathbf{A}\left(\begin{array}{c}
\beta_{1} \tau \\
\vdots \\
\beta_{n-1} \tau^{n-1}
\end{array}\right)=-c \tau^{n} \log \tau\left(\begin{array}{c}
1 \\
0 \\
\vdots \\
0
\end{array}\right)+\tau^{n} \mathbf{A}\left(\begin{array}{c}
\bar{R}_{n}(\tau) \\
\vdots \\
\bar{R}_{2 n-2}(\tau)
\end{array}\right)+\tau^{n} \log \tau\left(\begin{array}{c}
\gamma_{n} \\
\vdots \\
\gamma_{2 n-3} \\
0
\end{array}\right)
$$


After multiplication of both sides of (39) by $\mathbf{A}^{-1}$ we get

$$
\left(\begin{array}{c}
\beta_{1} \tau \\
\vdots \\
\beta_{n-1} \tau^{n-1}
\end{array}\right)=-c \tau^{n} \log \tau\left(\begin{array}{c}
a_{1,1} \\
\vdots \\
a_{n, 1}
\end{array}\right)+\tau^{n}\left(\begin{array}{c}
\bar{R}_{n}(\tau) \\
\vdots \\
\bar{R}_{2 n-2}(\tau)
\end{array}\right)+\tau^{n} \log \tau \mathbf{A}^{-1}\left(\begin{array}{c}
\gamma_{n} \\
\vdots \\
\gamma_{2 n-3} \\
0
\end{array}\right)
$$

Next, with (32) get

$$
\begin{aligned}
\alpha_{n-1} \tau^{n-1}= & -\sum_{j=1}^{n-1}\left(L_{n-1, j}+\log \tau H_{n-1, j}\right) \beta_{j} \tau^{j}-c \tau^{n} \log \tau H_{n-1, n} \\
& +\gamma_{n-1} \tau^{n} \log \tau-c \tau^{n} L_{n-1, n}-\tau^{n} \bar{P}_{n-1}(\tau),
\end{aligned}
$$

and for each $i=1, \ldots, n-2$,

$$
\begin{aligned}
\alpha_{i} \tau^{i}= & -\sum_{j=i+1}^{n-1} J_{i, j} \alpha_{j} \tau^{j}-\sum_{j=1}^{n-1}\left(L_{i, j}+\log \tau H_{i, j}\right) \beta_{j} \tau^{j} \\
& -c \tau^{n} \log \tau H_{i, n}+\gamma_{i} \tau^{n} \log \tau-c \tau^{n} L_{i, n}-\tau^{n} \bar{P}_{i}(\tau) .
\end{aligned}
$$

Recall that

$$
\begin{array}{rlrl}
\alpha_{i} & =\tau^{n-i} \log ^{2} \tau \bar{\alpha}_{i}(\gamma, \tau), & 0 \leq i \leq n-1, \\
\beta_{i}=\tau^{n-i} \log \tau \bar{\beta}_{i}(\gamma, \tau), & 1 \leq i \leq n-1 .
\end{array}
$$

Putting

$$
\left(\begin{array}{c}
\mathbb{V}_{1}(\gamma) \\
\vdots \\
\mathbb{V}_{n-2}(\gamma) \\
\mathbb{V}_{n-1}(\gamma)
\end{array}\right)=\mathbf{A}^{-1}\left(\begin{array}{c}
\gamma_{n} \\
\vdots \\
\gamma_{2 n-3} \\
0
\end{array}\right), \quad a_{i, 1}=a_{i}, \quad-c H_{i, n}=d_{i}
$$

by (40), this implies that

$$
\bar{\beta}_{i}(\gamma, \tau)=-c a_{i}-\log ^{-1} \tau \bar{R}_{n-1+i}(\tau)+\mathbb{V}_{i}(\gamma), \quad 1 \leq i \leq n-1,
$$

and

$$
\begin{aligned}
\bar{\alpha}_{n-1}(\gamma, \tau)= & -\sum_{j=1}^{n-1}\left(L_{n-1, j} \log ^{-1} \tau+H_{n-1, j}\right) \bar{\beta}_{j}(\gamma, \tau) \\
& -\left(c L_{n-1, n}+\bar{P}_{n-1}(\tau)\right) \log ^{-2} \tau+d_{n-1} \log ^{-1} \tau+\gamma_{n-1} \log ^{-1} \tau, \\
\bar{\alpha}_{i}(\gamma, \tau)= & -\sum_{j=i+1}^{n-1} J_{i, j} \bar{\alpha}_{j}(\gamma, \tau)-\sum_{j=1}^{n-1}\left(L_{i, j} \log ^{-1} \tau+H_{i, j}\right) \bar{\beta}_{j}(\gamma, \tau) \\
& -\left(c L_{i, n}+\bar{P}_{i}(\tau)\right) \log ^{-2} \tau+d_{i} \log ^{-1} \tau+\gamma_{i} \log ^{-1} \tau,
\end{aligned}
$$

for $0 \leq i \leq n-2$. Observe that (42), (43) together with (41) define a scaling in the parameter space. In the new parameters $(\gamma, \tau)$, the displacement function takes the form

$$
\Phi_{\Psi(\gamma, \tau)}(x)=\tau^{n} \log \tau\left(\gamma_{0}+\gamma_{1} x+\cdots+\gamma_{2 n-3} x^{2 n-3}+k(\gamma, \tau) x^{2 n-1}+\mathcal{O}\left(x^{2 n}\right)\right),
$$


where $k(0,0)=\tilde{k}(0) \neq 0$. By a rescaling of the form $\tilde{\gamma}_{i}=-\gamma_{i} /|k(\gamma, \tau)|$ and after removing the tildes, we get

$$
\Phi_{\Psi(\gamma, \tau)}(x)=-|k(\gamma, \tau)| \tau^{n} \log \tau\left(Q_{2 n-1}^{s, \pm}(x)+\mathcal{O}\left(x^{2 n}\right)\right) .
$$

This ends the proof of Theorem 1.

In the next section we prove Theorem 2. Though the idea is similar, there are differences in the computations that deserve to be mentioned.

2.2. Proof of Theorem 2. We now turn to the even case $k=2 n$. The strategy is the same as in the previous section. For $k=2 n$, the expression in (25) holds with $\alpha_{n}=c \neq 0$ constant and we have

$$
\Phi_{\alpha, \beta}(x)=\Lambda_{0}+\Lambda_{1} x+\cdots+\Lambda_{2 n-1} x^{2 n-1}+\Lambda_{2 n} x^{2 n}+\mathcal{O}\left(x^{2 n+1}\right),
$$

where

$$
\left(\begin{array}{c}
\Lambda_{0} \\
\vdots \\
\Lambda_{n-1}
\end{array}\right)=\mathbf{J}^{-}\left(\begin{array}{c}
\alpha_{0} \\
\alpha_{1} \tau \\
\vdots \\
\alpha_{n-1} \tau^{n-1}
\end{array}\right)+\left(\mathbf{L}^{-}+\log \tau \mathbf{H}^{-}\right)\left(\begin{array}{c}
\beta_{1} \tau \\
\vdots \\
\beta_{n} \tau^{n}
\end{array}\right)+\tau^{n+1} \log \tau\left(\begin{array}{c}
R_{0}(\tau) \\
R_{1}(\tau) \\
\vdots \\
R_{n-1}(\tau)
\end{array}\right),
$$

$$
\left(\begin{array}{c}
\Lambda_{n} \\
\vdots \\
\Lambda_{2 n-2} \\
\Lambda_{2 n-1}
\end{array}\right)=(\tilde{\mathbf{A}}+\log \tau \tilde{\mathbf{N}})\left(\begin{array}{c}
\beta_{1} \tau \\
\vdots \\
\beta_{n-1} \tau^{n-1} \\
\beta_{n} \tau^{n}
\end{array}\right)+c \tau^{n}\left(\begin{array}{c}
1 \\
0 \\
\vdots \\
0
\end{array}\right)+\tau^{n+1} \log \tau\left(\begin{array}{c}
R_{n}(\tau) \\
\vdots \\
R_{2 n-2}(\tau) \\
R_{2 n-1}(\tau)
\end{array}\right)
$$

and

$$
\left(\begin{array}{c}
\Lambda_{n+1} \\
\vdots \\
\Lambda_{2 n-1} \\
\Lambda_{2 n}
\end{array}\right)=\mathbf{T}\left(\begin{array}{c}
\beta_{1} \tau \\
\vdots \\
\beta_{n-1} \tau^{n-1} \\
\beta_{n} \tau^{n}
\end{array}\right)+\tau^{n+1} \log \tau\left(\begin{array}{c}
R_{n+1}(\tau) \\
\vdots \\
R_{2 n-1}(\tau) \\
R_{2 n}(\tau)
\end{array}\right)
$$

From (46), we get

$$
\Lambda_{n}=c \tau^{n}+\log \tau \beta_{n} \tau^{n}+\sum_{j=1}^{n} \tilde{A}_{n, j} \beta_{j} \tau^{j}+\tau^{n+1} \log \tau R_{n}(\tau),
$$

and for each integer $i=n+1, \ldots, 2 n-1$,

$$
\Lambda_{i}=\sum_{j=1}^{n} \tilde{A}_{i, j} \beta_{j} \tau^{j}+\tau^{n+1} \log \tau R_{i}(\tau)
$$

By Lemma 1, the matrix $\tilde{\mathbf{A}}$ is invertible. Putting

$$
\tilde{\mathbf{A}}^{-1}=\left(\tilde{a}_{i, j}\right)_{i=1, \ldots, n, j=1, \ldots, n},
$$


and after multiplication by $\tilde{\mathbf{A}}^{-1}$ on both sides of (46), we get

$$
\begin{aligned}
\tilde{\mathbf{A}}^{-1}\left(\begin{array}{c}
\Lambda_{n} \\
\vdots \\
\Lambda_{2 n-2} \\
\Lambda_{2 n-1}
\end{array}\right)= & \left(\mathbf{I d}+\log \tau \tilde{\mathbf{A}}^{-1} \tilde{\mathbf{N}}\right)\left(\begin{array}{c}
\beta_{1} \tau \\
\vdots \\
\beta_{n-1} \tau^{n-1} \\
\beta_{n} \tau^{n}
\end{array}\right) \\
& +c \tau^{n}\left(\begin{array}{c}
\tilde{a}_{1,1} \\
\vdots \\
\tilde{a}_{n-1,1} \\
\tilde{a}_{n, 1}
\end{array}\right)+\tau^{n+1} \log \tau \tilde{\mathbf{A}}^{-1}\left(\begin{array}{c}
R_{n}(\tau) \\
\vdots \\
R_{2 n-2}(\tau) \\
R_{2 n-1}(\tau)
\end{array}\right) .
\end{aligned}
$$

Observe that

$$
\tilde{\mathbf{A}}^{-1} \tilde{\mathbf{N}}=\left(\begin{array}{cccc}
0 & \ldots & 0 & \tilde{a}_{1,1} \\
0 & \ldots & 0 & \tilde{a}_{2,1} \\
\vdots & \ddots & \vdots & \vdots \\
0 & \ldots & 0 & \tilde{a}_{n, 1}
\end{array}\right)
$$

Now, we aim to find a scaled reparametrization of the form

$$
\Psi(\gamma, \tau)=(\alpha, \beta)
$$

linking $\gamma=\left(\gamma_{0}, \ldots, \gamma_{2 n-2}\right)$ and $\tau$ with $(\alpha, \beta)$. We require $\Psi$ to have the components

$$
\begin{gathered}
\alpha_{i}=\tau^{n-i} \bar{\alpha}_{i}(\gamma, \tau), \quad 0 \leq i \leq n-1, \\
\beta_{i}=\frac{\tau^{n-i}}{1+\tilde{a}_{n} \log \tau} \bar{\beta}_{i}(\gamma, \tau), \quad 1 \leq i \leq n,
\end{gathered}
$$

where $\tilde{a}_{n}$ is a constant, leading to

$$
\Phi_{\Psi(\gamma, \tau)}(x)=\tilde{f}(\tau)\left(\gamma_{0}+\gamma_{1} x+\cdots+\gamma_{2 n-2} x^{2 n-2}+k(\gamma, \tau) x^{2 n}+\mathcal{O}\left(x^{2 n+1}\right)\right) .
$$

We first determine $\tilde{f}(\tau)$ and show that the leading term $k(\gamma, \tau)$ is such that $k(0,0) \neq 0$. We then obtain the desired expression for $\Phi_{\Psi(\gamma, \tau)}$ by solving

$$
\Lambda_{i}=\tilde{f}(\tau) \gamma_{i}, \quad i=1, \ldots, 2 n-1
$$

To be more precise, we start by solving the equation

$$
\Lambda_{i}=0 \text { for all } i=n, \ldots, 2 n-1 \text {, and } \Lambda_{2 n} \neq 0 \text {. }
$$

With (48) and for each integer $i=1, \ldots, n$, we get

$$
\beta_{i} \tau^{i}+\log \tau \tilde{a}_{i, 1} \beta_{n} \tau^{n}+c \tilde{a}_{i, 1} \tau^{n}+\tau^{n+1} \log \tau Q_{n+i-1}(\tau)=0,
$$

where

$$
\left(\begin{array}{c}
Q_{n}(\tau) \\
\vdots \\
Q_{2 n-1}(\tau)
\end{array}\right)=\tilde{\mathbf{A}}^{-1}\left(\begin{array}{c}
R_{n}(\tau) \\
\vdots \\
R_{2 n-1}(\tau)
\end{array}\right)
$$


Again for each integer $i=n, \ldots, 2 n-1$,

$$
Q_{i}(\tau)=Q_{i, 0}+Q_{i, 1} \tau \log \tau+\cdots
$$

By putting $i=n$ in (49), we get

$$
\beta_{n}=-\frac{c \tilde{a}_{n, 1}+\tau \log \tau Q_{2 n-1}(\tau)}{1+\tilde{a}_{n, 1} \log \tau}
$$

and for each $i=1, \ldots, n-1$,

$$
\begin{gathered}
\beta_{i} \tau^{i}-\frac{c \tilde{a}_{n, 1} \tilde{a}_{i, 1} \tau^{n} \log \tau+\tilde{a}_{i, 1} \tau^{n+1} \log \tau^{2} Q_{2 n-1}(\tau)}{1+\tilde{a}_{n, 1} \log \tau} \\
=-c \tilde{a}_{i, 1} \tau^{n}-\tau^{n+1} \log \tau Q_{n+i-1}(\tau)
\end{gathered}
$$

which implies that

$$
\begin{aligned}
\beta_{i} \tau^{i}= & \frac{c \tilde{a}_{n, 1} \tilde{a}_{i, 1} \tau^{n} \log \tau+\tilde{a}_{i, 1} \tau^{n+1} \log \tau^{2} Q_{2 n-1}(\tau)}{1+\tilde{a}_{n, 1} \log \tau} \\
& +\frac{-c \tilde{a}_{i, 1} \tau^{n}-c \tilde{a}_{i, 1} \tilde{a}_{n, 1} \tau^{n} \log \tau}{1+\tilde{a}_{n, 1} \log \tau}-\tau^{n+1} \log \tau Q_{n+i-1}(\tau), \\
\beta_{i} \tau^{i}= & -\frac{c \tilde{a}_{i, 1} \tau^{n}}{1+\tilde{a}_{n, 1} \log \tau}+\frac{\tilde{a}_{i, 1} \tau^{n+1} \log ^{2} \tau Q_{2 n-1}(\tau)}{1+\tilde{a}_{n, 1} \log \tau}-\tau^{n+1} \log \tau Q_{n+i-1}(\tau) \\
= & -\frac{c \tilde{a}_{i, 1} \tau^{n}}{1+\tilde{a}_{n, 1} \log \tau}+\tau^{n+1} \log ^{2} \tau \frac{\bar{Q}_{i}(\tau)}{1+\tilde{a}_{n, 1} \log \tau},
\end{aligned}
$$

where $\bar{Q}_{i}$ is of the form

$$
\bar{Q}_{i}(\tau)=\bar{Q}_{i,-1} \log ^{-1} \tau+\bar{Q}_{i, 0}+\bar{Q}_{i, 1} \tau \log \tau+\cdots
$$

This also implies that

$$
\left(\begin{array}{c}
\beta_{1} \tau \\
\vdots \\
\beta_{n} \tau^{n}
\end{array}\right)=-\frac{c \tau^{n}}{1+\tilde{a}_{n, 1} \log \tau}\left(\begin{array}{c}
\tilde{a}_{1,1} \\
\vdots \\
\tilde{a}_{n, 1}
\end{array}\right)+\frac{\tau^{n+1} \log ^{2} \tau}{1+\tilde{a}_{n, 1} \log \tau}\left(\begin{array}{c}
\bar{Q}_{1}(\tau) \\
\vdots \\
\bar{Q}_{n}(\tau)
\end{array}\right)
$$

With (47) we get

$$
\left(\begin{array}{c}
\Lambda_{n+1}(0, \tau) \\
\vdots \\
\Lambda_{2 n}(0, \tau)
\end{array}\right)=-\frac{c \tau^{n}}{1+\tilde{a}_{n, 1} \log \tau} \mathbf{T}\left(\begin{array}{c}
\tilde{a}_{1,1} \\
\vdots \\
\tilde{a}_{n, 1}
\end{array}\right)+\frac{\tau^{n+1} \log ^{2} \tau}{1+\tilde{a}_{n, 1} \log \tau} \mathbf{T}\left(\begin{array}{c}
\bar{Q}_{1}(\tau) \\
\vdots \\
\bar{Q}_{n}(\tau)
\end{array}\right)
$$

By Lemma 1, both $\tilde{\mathbf{A}}^{-1}$ and $\mathbf{T}$ are invertible. It follows that

$$
\tilde{\mathbf{A}}^{-1}\left(\begin{array}{c}
1 \\
0 \\
\vdots \\
0
\end{array}\right)=\left(\begin{array}{c}
a_{1,1} \\
\vdots \\
a_{n, 1}
\end{array}\right) \neq 0 \text { and } \mathbf{T}\left(\begin{array}{c}
\tilde{a}_{1,1} \\
\vdots \\
\tilde{a}_{n, 1}
\end{array}\right) \neq 0
$$


thus $\left(\Lambda_{n+1}(0, \tau), \ldots, \Lambda_{2 n}(0, \tau)\right)$ does not vanish. Since for each $i=0, \ldots, 2 n-1$, $\Lambda_{i}=0$, this implies that

$$
\Lambda_{2 n}(0, \tau)=\bar{K}(\tau) \frac{\tau^{n}}{1+\tilde{a}_{n, 1} \log \tau}, \quad \text { with } \bar{K}(0) \neq 0
$$

We now develop the scaling. Putting

$$
\Lambda_{i}(\gamma, \tau)=\gamma_{i} \frac{\tau^{n}}{1+\tilde{a}_{n, 1} \log \tau}, \quad 0 \leq i \leq 2 n-2, \quad \Lambda_{2 n-1}(\gamma, \tau) \equiv 0
$$

with (48) it follows that, for each integer $i=1, \ldots, n$,

$$
\begin{aligned}
\beta_{i} \tau^{i} & +\log \tau \tilde{a}_{i, 1} \beta_{n} \tau^{n}+c \tilde{a}_{i, 1} \tau^{n}+\tau^{n+1} \log \tau Q_{n+i-1}(\tau) \\
= & \sum_{j=n}^{2 n-2} \tilde{a}_{i, j} \frac{\gamma_{j} \tau^{n}}{1+\tilde{a}_{n, 1} \log \tau}
\end{aligned}
$$

In particular we have

$$
\beta_{n} \tau^{n}+\log \tau \tilde{a}_{n, 1} \beta_{n} \tau^{n}=-c \tilde{a}_{n, 1} \tau^{n}+\sum_{j=n}^{2 n-2} \tilde{a}_{n, j} \frac{\gamma_{j} \tau^{n}}{1+\tilde{a}_{n, 1} \log \tau}-\tau^{n+1} \log \tau Q_{2 n-1}(\tau) .
$$

Thus

$$
\begin{aligned}
\beta_{n}= & -\frac{c \tilde{a}_{n, 1}}{\left(1+\tilde{a}_{n, 1} \log \tau\right)}+\frac{1}{\left(1+\tilde{a}_{n, 1} \log \tau\right)} \sum_{j=1}^{2 n-2} \tilde{a}_{i, j} \frac{\gamma_{j} \tau^{n}}{1+\tilde{a}_{n, 1} \log \tau} \\
& -\tau \log \tau Q_{2 n-1}(\tau) \frac{1}{1+\tilde{a}_{n, 1} \log \tau} .
\end{aligned}
$$

We now put

$$
\alpha_{i}=\tau^{n-i} \bar{\alpha}_{i}(\gamma, \tau), \quad \beta_{i}=\frac{\tau^{n-i}}{1+\tilde{a}_{n} \log \tau} \bar{\beta}_{i}(\gamma, \tau)
$$

Write

$$
\left(\begin{array}{c}
\mathbb{W}_{1}(\gamma) \\
\vdots \\
\mathbb{W}_{n-1}(\gamma) \\
\mathbb{W}_{n}(\gamma)
\end{array}\right)=\tilde{\mathbf{A}}^{-1}\left(\begin{array}{c}
\gamma_{n} \\
\vdots \\
\gamma_{2 n-2} \\
0
\end{array}\right)
$$

With (50), (51) and (52), it follows that

$$
\begin{aligned}
\bar{\beta}_{i}(\gamma, \tau)+\tilde{a}_{i, 1} \bar{\beta}_{n}(\gamma, \tau) \log \tau & +c \tilde{a}_{i, 1}\left(1+\tilde{a}_{n, 1} \log \tau\right) \\
& +\tau\left(1+\tilde{a}_{n, 1} \log \tau\right) \log \tau Q_{n+i-1}(\tau)=\sum_{j=n}^{2 n-2} \tilde{a}_{i, j} \gamma_{j}
\end{aligned}
$$


and we get

$$
\begin{aligned}
\bar{\beta}_{n}(\gamma, \tau)\left(1+\tilde{a}_{n, 1} \log \tau\right)= & -c \tilde{a}_{n, 1}\left(1+\tilde{a}_{n, 1} \log \tau\right) \\
& +\sum_{j=n}^{2 n-2} \tilde{a}_{i, j} \gamma_{j}-\tau\left(1+\tilde{a}_{n, 1} \log \tau\right) \log \tau Q_{2 n-1}(\tau), \\
\bar{\beta}_{n}(\gamma, \tau)= & -c \tilde{a}_{n}+\left(1+\tilde{a}_{n, 1} \log \tau\right)^{-1} \sum_{j=1}^{2 n-2} \tilde{a}_{n, j} \gamma_{j}-\tau \log \tau Q_{2 n-1}(\tau), \\
= & -c \tilde{a}_{n, 1}+\left(1+\tilde{a}_{n, 1} \log \tau\right)^{-1} \mathbb{W}_{n}(\gamma)-\tau \log \tau Q_{2 n-1}(\tau) .
\end{aligned}
$$

Moreover, with (53) and (54), for each $i=1, \ldots, n-1$, we get

$$
\begin{aligned}
\bar{\beta}_{i}(\gamma, \tau) & +\tilde{a}_{i, 1} \log \tau\left(-c \tilde{a}_{n, 1}+\left(1+\tilde{a}_{n, 1} \log \tau\right)^{-1} \mathbb{W}_{n}(\gamma)-\tau \log \tau Q_{2 n-1}(\tau)\right) \\
= & -c \tilde{a}_{i, 1}+\sum_{j=n}^{2 n-2} \tilde{a}_{i, 1} \gamma_{j}-\tau \log \tau Q_{n+i-1}(\tau) \\
- & c \tilde{a}_{i, 1} \tilde{a}_{n, 1} \log \tau-\tau \log ^{2} \tau \tilde{a}_{n, 1} Q_{n+i-1}(\tau), \\
\bar{\beta}_{i}(\gamma, \tau)= & -c \tilde{a}_{i}-\tilde{a}_{i} \log \tau\left(1+\tilde{a}_{n} \log \tau\right)^{-1} \mathbb{W}_{n}(\gamma)+\mathbb{W}_{i}(\gamma)-\tau \log \tau Q_{n+i-1}(\tau) \\
& -\tau \log ^{2} \tau \tilde{a}_{n} Q_{n+i-1}(\tau)+\tilde{a}_{i} \tau \log ^{2} \tau Q_{2 n-1}(\tau),
\end{aligned}
$$

where $\tilde{a}_{i}=\tilde{a}_{i, 1}$. Now with (45), we get

$$
\begin{aligned}
\alpha_{n-1} \tau^{n-1}= & \gamma_{n-1} \frac{\tau^{n}}{1+\tilde{a}_{n, 1} \log \tau}-c L_{n-1,0} \tau^{n} \\
& -\sum_{j=1}^{n}\left(L_{n-1, j}+\log \tau H_{n-1, j}\right) \beta_{j} \tau^{j}-\tau^{n+1} \log \tau R_{n-1}(\tau),
\end{aligned}
$$

thus

$$
\begin{aligned}
\bar{\alpha}_{n-1}(\gamma, \tau)= & -c \ell_{n}+\gamma_{n-1}\left(1+\tilde{a}_{n} \log \tau\right)^{-1} \\
& -\left(\frac{\log \tau}{1+\tilde{a}_{n} \log \tau}\right) \sum_{j=1}^{n}\left(L_{n-1, j} \log ^{-1} \tau+H_{n-1, j}\right) \bar{\beta}_{j}(\gamma, \tau) \\
& -\tau \log \tau R_{n-1}(\tau),
\end{aligned}
$$

where $\ell_{i}=L_{i, 0}$ and for each $i=1, \ldots, n-2$,

$$
\begin{aligned}
\alpha_{i} \tau^{i}= & -c \ell_{i} \tau^{n}-\sum_{j=i+1}^{n-1} J_{i, j} \alpha_{j} \tau^{j}+\gamma_{i} \tau^{n}\left(1+\tilde{a}_{n, 1} \log \tau\right)^{-1} \\
& -\sum_{j=1}^{n}\left(L_{i, j}+\log \tau H_{i, j}\right) \beta_{j} \tau^{j}-\tau^{n+1} \log \tau R_{i}(\tau),
\end{aligned}
$$

thus

$$
\begin{aligned}
\bar{\alpha}_{i}(\gamma, \tau)= & -c \ell_{i}-\sum_{j=i+1}^{n-1} J_{i, j} \bar{\alpha}_{j}(\gamma, \tau)+\gamma_{i}\left(1+\tilde{a}_{n, 1} \log \tau\right)^{-1} \\
& -\left(\frac{\log \tau}{1+\tilde{a}_{n} \log \tau}\right) \sum_{j=1}^{n}\left(L_{i, j} \log ^{-1} \tau+H_{i, j}\right) \bar{\beta}_{j}(\gamma, \tau)-\tau \log \tau R_{i}(\tau) .
\end{aligned}
$$


Observe that the expressions given in (54), (55) and (56) together with (52) define a scaling. In the new parameters $(\gamma, \tau)$, the displacement function takes the form

$$
\Phi_{\Psi(\gamma, \tau)}(x)=\frac{\tau^{n}}{1+\tilde{a}_{n} \log \tau}\left(\gamma_{0}+\gamma_{1} x+\cdots+\gamma_{2 n-2} x^{2 n-2}+k(\gamma, \tau) x^{2 n}+\mathcal{O}\left(x^{2 n+1}\right)\right),
$$

where $k(0,0)=\bar{K}(0) \neq 0$. By a rescaling of the form $\tilde{\gamma}_{i}=-\gamma_{i} /|k(\gamma, \tau)|$ and after removing the tildes, we get

$$
\Phi_{\Psi(\gamma, \tau)}(x)=-\frac{|k(\gamma, \tau)| \tau^{n}}{1+\tilde{a}_{n} \log \tau}\left(Q_{2 n}^{s, \pm}(x)+\mathcal{O}\left(x^{2 n+1}\right)\right) .
$$

This ends the proof of Theorem 2 .

2.3. Proof of Theorems 3 and 4. To construct the scaling, we first recall that

$$
\begin{aligned}
B_{\lambda, \varepsilon}(u)= & \alpha_{0}+\beta_{1}\left[u \omega+\varepsilon \eta_{1}(u, u \omega)\right]+\alpha_{1}\left[u+\varepsilon \mu_{1}(u, u \omega)\right]+\cdots \\
& +\alpha_{n-1}\left[u^{n-1}+\varepsilon \mu_{n-1}(u, u \omega)\right]+\beta_{n}\left[u^{n} \omega+\varepsilon \eta_{n}(u, u \omega)\right]+\Psi_{n}(u, \lambda, \varepsilon) .
\end{aligned}
$$

For simplicity we assume that $\beta_{n}=c \neq 0$ and we are treating an unfolding of odd codimension. In the even-codimension case, the proof is completely similar.

Let $\tau>0$, and put $u=\tau(1+x)$ where $x$ is close to 0 . Recall that

$$
\omega(u)=\frac{u^{\varepsilon \tilde{\beta}}-1}{\varepsilon \tilde{\beta}} .
$$

It follows that

$$
\begin{aligned}
\omega[\tau(1+x)] & =\frac{1}{\varepsilon \tilde{\beta}}\left(\tau^{\varepsilon \tilde{\beta}}(1+x)^{\varepsilon \tilde{\beta}}-(1+x)^{\varepsilon \tilde{\beta}}+(1+x)^{\varepsilon \tilde{\beta}}-1\right) \\
& =\omega(\tau)(1+x)^{\varepsilon \tilde{\beta}}+\omega(1+x) \\
& =\omega(\tau)\left(1+\varepsilon \mathcal{A}_{1}(x, \varepsilon)\right)+\log (1+x)+\varepsilon \mathcal{A}_{2}(x, \varepsilon),
\end{aligned}
$$

where both $\mathcal{A}_{1}$ and $\mathcal{A}_{2}$ are analytic functions. Consider the maps

$$
u \mapsto G_{j}(u)=\beta_{j}\left(u^{j} \omega(u)+\varepsilon \eta_{j}(u, u \omega(u))\right), \quad 1 \leq j \leq n-1,
$$

and

$$
u \mapsto F_{i}(u)=\alpha_{i}\left(u^{i}+\varepsilon \mu_{i}(u, u \omega)\right), \quad 0 \leq i \leq n-1 .
$$

For all integers $i, j$ under consideration, we have

$$
\begin{aligned}
G_{j}(\tau(1+x))= & \beta_{j}\left[\tau^{j}(1+x)^{j} \omega(\tau(1+x))\right. \\
& \left.+\varepsilon \eta_{j}[\tau(1+x),(\tau(1+x) \omega(\tau(1+x)))]\right], \\
F_{i}(\tau(1+x))= & \alpha_{i}\left[\tau^{i}(1+x)^{i}+\varepsilon \mu_{i}[\tau(1+x),(\tau(1+x) \omega(\tau(1+x)))]\right] .
\end{aligned}
$$

With (57) and knowing that

$$
\begin{gathered}
\eta_{j}(u, u \omega(u))=\eta_{i, 0} u^{j}+\cdots, \quad 1 \leq j \leq n-1, \\
\mu_{i}(u, u \omega(u))=\mu_{i, 0} u^{i+1} \omega(u)+\cdots, \quad 0 \leq i \leq n-1,
\end{gathered}
$$


it follows that

$$
\begin{aligned}
G_{j}(\tau(1+x))= & \beta_{j} \tau^{j}\left[(1+x)^{j} \omega(\tau)\left(1+\varepsilon \mathcal{A}_{1}(x, \varepsilon)+\log (1+x)+\varepsilon \mathcal{A}_{2}(x, \varepsilon)\right)\right. \\
& \left.+\varepsilon \mathcal{C}_{j}(x, \varepsilon, \tau, \tau \omega(\tau))\right], \quad j=1, \ldots, n-1, \\
F_{i}(\tau(1+x))= & \alpha_{i} \tau^{i}\left[(1+x)^{i}+\varepsilon \mathcal{D}_{i}(x, \varepsilon, \tau, \tau \omega(\tau))\right], \quad i=0, \ldots, n-1,
\end{aligned}
$$

where $\mathcal{C}_{j}$ and $\mathcal{D}_{i}$ are smooth in $x$ and $\varepsilon$, polynomial in $\tau$ and $\omega^{n}(\tau)$ and satisfy

$$
\mathcal{C}_{j}=C_{j, 0}+\cdots, \quad \mathcal{D}_{i}=D_{i, 0} \tau \omega^{i+1}(\tau)+\cdots .
$$

Write $B_{\lambda, \varepsilon}(\tau(1+x))=\Phi_{\alpha, \beta}(x)$. We get

$$
\Phi_{\alpha, \beta}(x)=\Lambda_{0}+\Lambda_{1} x+\cdots+\Lambda_{2 n-1} x^{2 n-2}+\Lambda_{2 n-1} x^{2 n-1}+\mathcal{O}\left(x^{2 n}\right) .
$$

From (58) and (59), there exist matrices

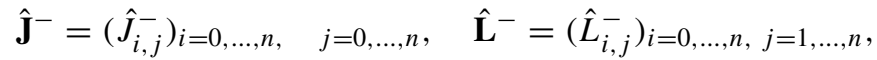

$$
\begin{aligned}
& \hat{\mathbf{H}}^{-}(\varepsilon)=\left(\hat{H}_{i, j}^{-}(\varepsilon)\right)_{i=0, \ldots, n, j=1, \ldots, n}, \quad \hat{\mathbf{T}}=\left(T_{i, j}\right)_{i=n+1, \ldots, 2 n, j=1, \ldots, n},
\end{aligned}
$$

and $\check{\mathbf{N}}$ with entries that are polynomial in $\tau$ and $\omega^{n}(\tau)$ and analytic in $\varepsilon$ such that

$$
\begin{gathered}
\hat{J}_{i, j}^{-}=J_{i, j}^{-}+\cdots, \quad \hat{H}_{i, j}^{-}=H_{i, j}^{-}+\cdots, \quad \hat{L}_{i, j}^{-}=L_{i, j}^{-}+\cdots, \\
\hat{T}_{i, j}=T_{i, j}+\cdots, \quad \check{N}_{i, j}=\tilde{N}_{i, j}+\cdots,
\end{gathered}
$$

and such that

$$
\begin{aligned}
\left(\begin{array}{c}
\Lambda_{0} \\
\vdots \\
\Lambda_{n-1}
\end{array}\right)= & \hat{\mathbf{J}}^{-}\left(\begin{array}{c}
\alpha_{0} \\
\alpha_{1} \tau \\
\vdots \\
\alpha_{n-1} \tau^{n-1}
\end{array}\right)+\omega(\tau) \hat{\mathbf{H}}^{-}\left(\begin{array}{c}
\beta_{1} \tau \\
\vdots \\
\beta_{n-1} \tau^{n-1} \\
c \tau^{n}
\end{array}\right) \\
& +\hat{\mathbf{L}}^{-}\left(\begin{array}{c}
\beta_{1} \tau \\
\vdots \\
\beta_{n-1} \tau^{n-1} \\
c \tau^{n}
\end{array}\right)+\left(\begin{array}{c}
\hat{P}_{0}(\tau, \varepsilon) \\
\vdots \\
\hat{P}_{n-2}(\tau, \varepsilon) \\
\hat{P}_{n-1}(\tau, \varepsilon)
\end{array}\right)
\end{aligned}
$$

where for each integer $i=0, \ldots, n-1$,

$$
\hat{P}_{i}(\tau, \varepsilon)=\tau^{n} \bar{P}_{i}(\tau, \varepsilon)=\tau^{n}\left[\bar{P}_{i}(0, \varepsilon)+\cdots\right] .
$$

Define also the following two matrices:

$$
\begin{aligned}
& \check{\mathbf{A}}=\left(\check{A}_{i, j}\right)_{i=n, \ldots, 2 n-1, j=1, \ldots, n}, \quad \check{A}_{i, j}=\hat{T}_{i, j} \text { if } i>n, \quad \check{A}_{n, j}=\hat{L}_{n, j}^{-},
\end{aligned}
$$

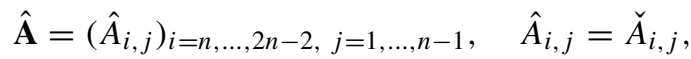

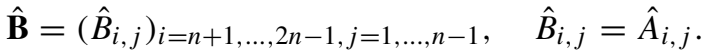

From Lemma 1, these three matrices remain invertible for $\varepsilon$ sufficiently small. We get

$$
\left(\begin{array}{c}
\Lambda_{n} \\
\vdots \\
\Lambda_{2 n-2} \\
\Lambda_{2 n-1}
\end{array}\right)=(\check{\mathbf{A}}+\log \tau \check{\mathbf{N}})\left(\begin{array}{c}
\beta_{1} \tau \\
\vdots \\
\beta_{n-1} \tau^{n-1} \\
c \tau^{n}
\end{array}\right)+\left(\begin{array}{c}
P_{n}(\tau, \varepsilon) \\
\vdots \\
P_{2 n-2}(\tau, \varepsilon) \\
P_{2 n-1}(\tau, \varepsilon)
\end{array}\right),
$$


where for each integer $i=n, \ldots, 2 n-1$,

$$
P_{i}(\tau, \varepsilon)=\tau^{n} \bar{P}_{i}(\tau, \varepsilon)=\tau^{n}\left[\bar{P}_{i}(\tau, \varepsilon)+\cdots\right] .
$$

We follow the same strategy as in the previous section. We claim that for each integer $i=$ $1, \ldots, n-1$, there exist maps $\hat{R}_{n-1+i}(\tau, \varepsilon), \hat{\mathbb{V}}_{i}(\tau, \gamma, \varepsilon)$ and coefficients $\hat{a}_{i}(\tau, \varepsilon), \hat{d}_{i}(\tau, \varepsilon)$ which are smooth in $\varepsilon$ and $\tau \omega^{n}(\tau),\left(\hat{\mathbb{V}}_{i}\right.$ is linear in $\left.\gamma\right)$, such that

$$
\begin{aligned}
\hat{a}_{i}(\tau, \varepsilon) & =a_{i}+\cdots, \quad \hat{d}_{i}(\tau, \varepsilon)=d_{i}+\cdots, \\
\hat{R}_{n-1+i}(\tau, \varepsilon) & =\bar{R}_{n-1+i}+\cdots, \quad \hat{\mathbb{V}}_{i}(\tau, \gamma, \varepsilon)=\mathbb{V}_{i}+\cdots,
\end{aligned}
$$

and such that by putting

$$
\begin{gathered}
\beta_{i}=\tau^{n-i} \omega(\tau) \bar{\beta}_{i}(\tau, \gamma, \varepsilon), \quad \alpha_{i}=\bar{\alpha}_{i}(\tau, \gamma, \varepsilon) \tau^{n-i} \omega^{2}(\tau) \\
\bar{\beta}_{i}(\tau, \gamma, \varepsilon)=-c \hat{a}_{i}(\tau, \varepsilon)-\omega^{-1}(\tau) \hat{R}_{n-1+i}(\tau, \varepsilon)+\hat{\mathbb{V}}_{i}(\tau, \gamma, \varepsilon)
\end{gathered}
$$

and

$$
\begin{aligned}
\bar{\alpha}_{n-1}(\tau, \gamma, \varepsilon)= & -\sum_{j=1}^{n-1}\left(\hat{L}_{n-1, j}(\tau, \varepsilon) \omega^{-1}(\tau)+\hat{H}_{n-1, j}(\tau, \varepsilon)\right) \bar{\beta}_{j}(\tau, \gamma, \varepsilon) \\
& -\left(c \hat{L}_{n-1, n}(\tau, \varepsilon)+\check{P}_{n-1}(\tau, \varepsilon)\right) \omega^{-2}(\tau) \\
& +\hat{d}_{n-1}(\tau, \varepsilon) \omega^{-1}(\tau)+\gamma_{n-1} \omega^{-1}(\tau), \\
\bar{\alpha}_{i}(\tau, \gamma, \varepsilon)= & -\sum_{j=i+1}^{n-1} \hat{J}_{i, j}(\tau, \varepsilon) \bar{\alpha}_{j}(\tau, \gamma, \varepsilon) \\
& -\sum_{j=1}^{n-1}\left(\hat{L}_{i, j}(\tau, \varepsilon) \omega^{-1}(\tau)+\hat{H}_{i, j}(\tau, \varepsilon)\right) \bar{\beta}_{j}(\tau, \gamma, \varepsilon) \\
& -\left(c \hat{L}_{i, n}(\tau, \varepsilon)+\check{P}_{i}(\tau, \varepsilon)\right) \omega^{-2}(\tau)+\hat{d}_{i}(\tau, \varepsilon) \omega^{-1}(\tau) \\
& +\gamma_{i} \omega^{-1}(\tau),
\end{aligned}
$$

for $i=0, \ldots, n-2$, we get

$$
\Phi_{\psi(\gamma, \tau)}(x)=\tau^{n} \omega(\tau)\left(\gamma_{0}+\gamma_{1}+\cdots+\gamma_{2 n-3} x^{2 n-3}+\mathcal{K}(\tau, \gamma, \varepsilon) x^{2 n-1}+\mathcal{O}\left(x^{2 n}\right)\right),
$$

where $\mathcal{K}(0,0,0) \neq 0$.

Acknowledgements. We thank Pavao Marděsic, Carles Simó, Floris Takens, Renato Vitolo and Florian Wagener for their help and stimulating discussions.

\section{REFERENCES}

[1] A. A. Andronov, E. A. Leontovich, I. I. Gordon and A. G. Maier. Theory of Dynamical Systems on a Plane. Israel Program of Scientific Translations, Jerusalem, 1971.

[2] V. I. Arnol'd. Proof of a theorem by A. N. Kolmogorov on the persistence of quasi-periodic motions under small perturbations of Hamiltonian. Russian Math. Surveys 18(5) (1963), 9-36. 
[3] V. I. Arnol'd. Geometrical Methods in the Theory of Ordinary Differential Equations. Springer, Berlin, 1983.

[4] V. Bogdanov. Versal deformations of a singular point of a vector field on the plane in the case of two zeros eigenvalues. Trudy Seminara Petrovvskogo 2 (1976), 37-65; Selecta Math. 1 (1981), 389-491.

[5] Th. Broecker and L. Lander. Differential Germs and Catastrophes (London Mathematical Society Lecture Note Series, 17). Cambridge University Press, Cambridge, 1975.

[6] H. W. Broer, F. Dumortier, S. J. van Strien and F. Takens. Structures in Dynamics, Finite Dimensional Deterministic Studies (Studies in Mathematical Physics, 2). North-Holland, Amsterdam, 1991.

[7] H. W. Broer, H. Hanßmann and J. You. Bifurcations of normally parabolic tori in Hamiltonian systems. Nonlinearity 18 (2005), 1735-1769.

[8] H. W. Broer, H. Hanßmann and J. You. Umbilical torus bifurcations in Hamiltonian systems. J. Differential Equations 222 (2006), 233-262.

[9] H. W. Broer, J. Hoo and V. Naudot. Normal linear stability of quasi periodic tori. Preprint, University of Groningen, 2004.

[10] H. W. Broer and G. B. Huitema. Unfoldings of quasi-periodic tori in reversible systems. J. Dynam. Differential Equations 7(1) (1995), 191-212.

[11] H. W. Broer, G. B. Huitema and M. B. Sevryuk. Quasi Periodic Motions in Families of Dynamical Systems (Lecture Notes in Mathematics, 1645). Springer, Berlin, 1996.

[12] H. W. Broer, G. B. Huitema, F. Takens and B. L. J. Braaksma. Unfoldings and bifurcations of quasi periodic tori. Mem. Amer. Math. Soc. 83(421) (1990), 1-175.

[13] H. W. Broer, V. Naudot, R. Roussarie and F. O. O. Wagener. On persistence of diophantine tori for nearly Hamiltonian systems with a homoclinic orbit. In preparation.

[14] H. W. Broer and R. Roussarie. Exponential confinement of chaos in the bifurcation set of real analytic diffeomorphisms. Global Analysis of Dynamical Systems, Festschrift Dedicated to Floris Takens for his 60th birthday. Eds. H. W. Broer, B. Krauskopf and G. Vegter. IOP Publishing, Bristol, 2001, pp. 167-210.

[15] H. W. Broer, R. Roussarie and C. Simó. A numerical survey on the Takens-Bogdanov bifurcation for diffeomorphism. Eur. Conf. on Iteration Theory, 89. Eds. C. Mira, N. Netzer, C. Simó and G. Targonski. Word Scientific, Singapore, 1992, pp. 320-334.

[16] H. W. Broer, R. Roussarie and C. Simó. On the Bogdanov-Takens bifurcation for planar diffeomorphisms. Int. Conf. on Differential Equations, 1, 2 (Barcelona, 1991). World Scientific, River Edge, NJ, 1993, pp. 81-92.

[17] H. W. Broer, R. Roussarie and C. Simó. Invariant circles in the Bogdanov-Takens bifurcation for diffeomorphisms. Ergod. Th. \& Dynam. Sys. 16 (1996), 1147-1172.

[18] H. W. Broer, C. Simó and J. C. Tatjer. Towards global models near homoclinic tangencies of dissipative diffeomorphisms. Nonlinearity 11 (1989), 667-771.

[19] A. Chenciner. Bifurcation des points fixes elliptiques. I. Publ. Math. Inst. Hautes Études Sci. 61 (1985), 67-127.

[20] A. Chenciner. Bifurcation des points fixes elliptiques. II: Orbites périodiques et ensembles de Cantor invariants. Invent. Math. 80(1) (1985), 81-106.

[21] A. Chenciner. Bifurcation des points fixes elliptiques. III: Orbites périodiques de 'petites' périodes et élimination résonante des couples de courbes invariantes. Publ. Math. Inst. Hautes Études Sci. 66 (1988), $5-91$.

[22] F. Dumortier. Singularities of vector fields on the plane. J. Differential Equations 23(2) (1977), 53-166.

[23] F. Dumortier, R. Roussarie and J. Sotomayor. Generic 3-parameter families of vector field on the plane, unfolding a singularity with nilpotent linear part. The cusp case of codimension 3. Ergod. Th. \& Dynam. Sys. 7 (1987), 375-413.

[24] F. Dumortier, R. Roussarie, J. Sotomayor and H. Zoladek. Bifurcations of Planar Vector Fields (Lecture Notes in Mathematics, 1480). Springer, Berlin, 1991.

[25] F. Dumortier and C. Rousseau. Cubic Liénard equations with linear damping. Nonlinearity 3 (1990), $1015-1039$.

[26] C. G. Gibson. Singular Points of Smooth Mappings (Research Notes in Mathematics, 25). Pitman, London, 1979.

[27] M. Golubitsky and V. Guillemin. Stable Mappings and Their Singularities. Springer, Berlin, 1973.

[28] A. Khibnik, B. Krauskopf and C. Rousseau. Global study of a family of cubic Liénard equation. Nonlinearity 11 (1998), 1505-1519.

[29] Yu. A. Kuznetsov. Elements of Applied Bifurcation Theory. Springer, Berlin, 1995. 
[30] S. Łojasiewicz. Whitney fields and the Malgrange-Mather Preparation Theorem. Proceedings of Liverpool Singularities-Symposium, I (1969/70) (Lecture Notes in Mathematics, 192). Springer, Berlin, 1971, pp. 106-115.

[31] C. Z. Li and C. Rousseau. A system with three limit cycles appearing in a Hopf bifurcation and dying in a homoclinic bifurcation: the cusp of order 4. J. Differential Equations 79(1) (1989), 132-167.

[32] B. Malgrange. Ideals of Differentiable Functions. Oxford University Press, Oxford, 1966.

[33] P. Marděsic. The number of limit cycles of polynomial deformations of a Hamiltonian vector field. Ergod. Th. \& Dynam. Sys. 10 (1990), 523-529.

[34] P. Marděsic. Déploiement versel du cusp d'order $n$. Doctorat, l'Université de Bourgogne, 1992.

[35] P. Marděsic. Chebychev systems and the versal unfolding of the cusp of order $n$. Travaux en Cours. Hermann, Paris, 1994, pp. 1-120.

[36] J. Mather. Stability of $C^{\infty}$ mappings I. The division theorem. Ann. of Math. (2) 89 (1968), $254-291$.

[37] L. Mora and M. Viana. Abundance of strange attractors. Acta Math. 171 (1993), 1-71.

[38] J. Moser. Convergent series expansions for quasi-periodic motions. Math. Ann. 169 (1967), 136-176.

[39] R. Narasimhan. Introduction to the Theory of Analytic Spaces (Lecture Notes in Mathematics, 25). Springer, Berlin, 1966.

[40] A. I. Neishtadt. The separation of motions in systems with rapidly rotation phase. J. Appl. Math. Mech. 48 (1976), 133-139.

[41] J. Palis and W. de Melo. Geometric Theory of Dynamical Systems, An Introduction. Springer, Berlin, 1982.

[42] J. Palis and F. Takens. Hyperbolicity and Sensitive Chaotic Dynamics at Homoclinic Bifurcations. Fractal Dimensions and Infinitely Many Attractors. Cambridge University Press, Cambridge, 1993.

[43] T. Poston and I. N. Stewart. Catastrophe Theory and its Applications. Pitman, London, 1978.

[44] R. Roussarie. On the number of limit cycles which appear by perturbation of a separatrix loop of planar vector fields. Bol. Soc. Brasol Mat. 17(2) (1985), 67-101.

[45] F. Takens. A nonstabilizable jet of a singularity of a vector field. Dynamical Systems (Proc. Symp. Univ. Bahia. Salvador, 1971). Academic Press, New York, 1973, pp. 583-597.

[46] F. Takens. Singularities of vector fields. Publ. Math. Inst. Hautes Études Sci. 43 (1974), 47-100.

[47] F. Takens. Unfolding of certain singularities of vector fields: generalized Hopf bifurcations. J. Dynam. Differential Equations 14 (1974), 476-93.

[48] F. Takens. Forced oscillations and bifurcations. Application of global analysis I. Commun. Math. Inst. Univ. Utrecht 3 (1974), 1-59. Reprinted in Eds. H. W. Broer, B. Krauskopf and G. Vegter. Global Analysis of Dynamical Systems, Festschrift Dedicated to Floris Takens for his 60th birthday. IOP Publishing, Bristol, 2001, pp. 1-61.

[49] R. Thom. Structural Stability and Morphogenesis. Transl. D. H. Fowler. Benjamin-Addison Wesley, New York, 1975. (Translation of Stabilité Structurelle et Morphogénèse with additional material.)

[50] R. Vitolo. Bifurcations of attractors in $3 \mathrm{D}$ diffeomorphisms. A study in experimental mathematics. $P h D$ Thesis, University of Groningen, 2003.

[51] F. O. O. Wagener. On the quasi-periodic $d$-fold degenerate bifurcation. CeNDEF Preprint, 2003.

[52] F. O. O. Wagener. On Moser's modifying terms theorem and its application to quasi-periodic bifurcation theory. CeNDEF Preprint, 2003.

[53] A. E. R. Woodcock and T. Poston. A Geometrical Study of the Elementary Catastrophes (Lecture Notes in Mathematics, 373). Springer, Berlin, 1974. 